\title{
LYN- and AIRE-mediated tolerance checkpoint defects synergize to trigger organ-specific autoimmunity
}

\author{
Irina Proekt, ${ }^{1}$ Corey N. Miller, ${ }^{2}$ Marion Jeanne, ${ }^{3}$ Kayla J. Fasano, ${ }^{2}$ James J. Moon, ${ }^{4}$ Clifford A. Lowell, ${ }^{5}$ Douglas B. Gould, ${ }^{3}$ \\ Mark S. Anderson, ${ }^{2}$ and Anthony L. DeFranco ${ }^{1}$ \\ 'Department of Microbiology and Immunology, ${ }^{2}$ Diabetes Center, ${ }^{3}$ Departments of Ophthalmology and Anatomy, Institute for Human Cenetics, UCSF, San Francisco, California, USA. ${ }^{4}$ Center for Immunology \\ and Inflammatory Diseases and Division of Pulmonary and Critical Care Medicine, Massachusetts Ceneral Hospital and Harvard Medical School, Boston, Massachusetts, USA. ${ }^{5}$ Department of Laboratory \\ Medicine, UCSF, San Francisco, California, USA.
}

\begin{abstract}
Studies of the genetic factors associated with human autoimmune disease suggest a multigenic origin of susceptibility; however, how these factors interact and through which tolerance pathways they operate generally remain to be defined. One key checkpoint occurs through the activity of the autoimmune regulator AIRE, which promotes central T cell tolerance. Recent reports have described a variety of dominant-negative AIRE mutations that likely contribute to human autoimmunity to a greater extent than previously thought. In families with these mutations, the penetrance of autoimmunity is incomplete, suggesting that other checkpoints play a role in preventing autoimmunity. Here, we tested whether a defect in LYN, an inhibitory protein tyrosine kinase that is implicated in systemic autoimmunity, could combine with an Aire mutation to provoke organ-specific autoimmunity. Indeed, mice with a dominant-negative allele of Aire and deficiency in LYN spontaneously developed organ-specific autoimmunity in the eye. We further determined that a small pool of retinal protein-specific T cells escaped thymic deletion as a result of the hypomorphic Aire function and that these cells also escaped peripheral tolerance in the presence of LYN-deficient dendritic cells, leading to highly destructive autoimmune attack. These findings demonstrate how 2 distinct tolerance pathways can synergize to unleash autoimmunity and have implications for the genetic susceptibility of autoimmune disease.
\end{abstract}

\section{Introduction}

In most individuals, a diversity of tolerance mechanisms work together to prevent autoimmune disease by limiting the activity of self-reactive lymphocytes (1). Immune tolerance requires integrity of multiple checkpoints within both innate and adaptive branches of the immune system, but how such checkpoints fail in some individuals, leading to particular autoimmune phenotypes, remains poorly understood. Strikingly, most autoimmune diseases exhibit a high degree of genetic susceptibility, and in most cases this susceptibility is multigenic in origin $(2,3)$. In spite of this knowledge, little is known about how multiple genes interact to cause disease and which tolerance pathways are compromised.

Humans that harbor a complete defect in the autoimmune regulator $(A I R E)$ gene develop a multiorgan autoimmune syndrome called autoimmune polyglandular syndrome type 1 (APS1) $(4,5)$. AIRE promotes $T$ cell tolerance by driving the expression of a wide array of tissue-specific self-antigens (TSAs) in the thymus and therefore is critical for central tolerance of $\mathrm{T}$ cells recognizing these TSAs (6). In addition to rare individuals with autoimmunity and a complete absence of AIRE, a family was identified

Authorship note: M.S. Anderson and A.L. DeFranco are co-senior authors. Conflict of interest: M. Jeanne is an employee of Genentech Inc., a member of the Roche Group, and has an equity interest in Roche through an employee plan. M.S. Anderson's spouse is a shareholder of Medtronic and Merck. A.L. DeFranco is a consultant and expert witness for law firms representing Regeneron Inc.

Submitted: September 3, 2015; Accepted: July 22, 2016.

Reference information: / Clin Invest. 2016;126(10):3758-3771. doi:10.1172/JCI84440. with an AIRE mutation and dominantly inherited autoimmune susceptibility $(7,8)$. A recent study indicates that individuals with dominantly acting alleles of AIRE may be more widespread than initially thought (7). Individuals with dominant AIRE mutations develop autoimmunity that is less penetrant and has later onset compared with individuals with complete AIRE deficiency. These clinical observations are consistent with hypomorphic AIRE function in individuals with 1 dominant-negative AIRE allele and 1 normal allele, and suggest that additional genetic alterations, perhaps affecting other tolerance checkpoints, may contribute to disease onset in such individuals.

Whereas defects in AIRE function lead to organ-specific autoimmune diseases in humans and mice, defects in Lyn lead to a severe lupus-like systemic autoimmunity in mice $(9,10)$. LYN is an Src-family protein tyrosine kinase that is expressed by B cells, myeloid cells, and dendritic cells (DCs), but not by T cells. It has a unique role of enabling function of inhibitory receptors that have immunoreceptor tyrosine-based inhibitory motifs (ITIMs) in their cytoplasmic domains by phosphorylating the ITIMs (9). Selective deletion in mice of Lyn in B cells or in DCs leads to systemic autoimmunity, the former being especially important for production of antinuclear antibodies and the latter being important for increased inflammation (11-13). Although single-nucleotide polymorphisms (SNPs) near the $L Y N$ gene have been associated with systemic lupus erythematosus (SLE) in human genome-wide association studies, it is unclear whether these SNPs affect LYN expression or function $(14,15)$. There is, however, strong evidence that genetic variation related to 2 inhibitory receptors downstream of LYN can 
A
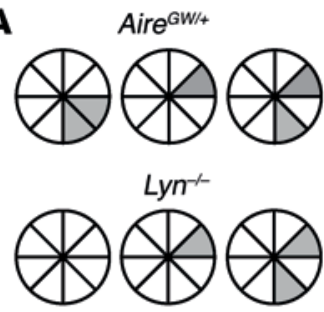
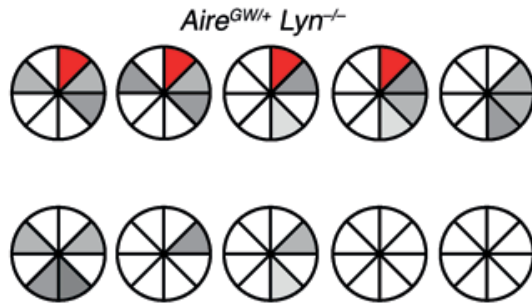
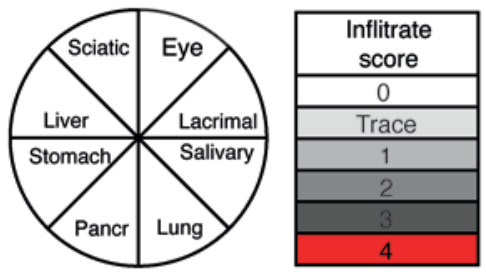

B
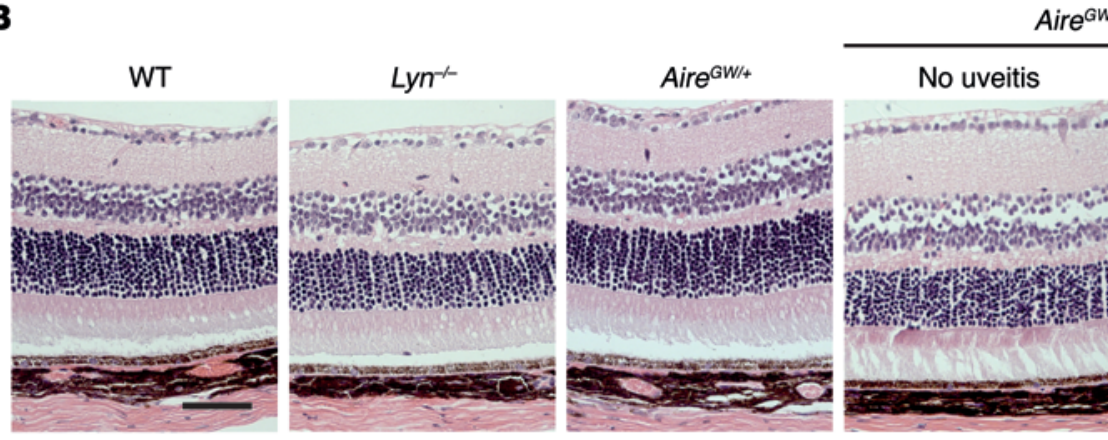

Aire ${ }^{G W /+} \mathrm{Lyn}^{-1}$
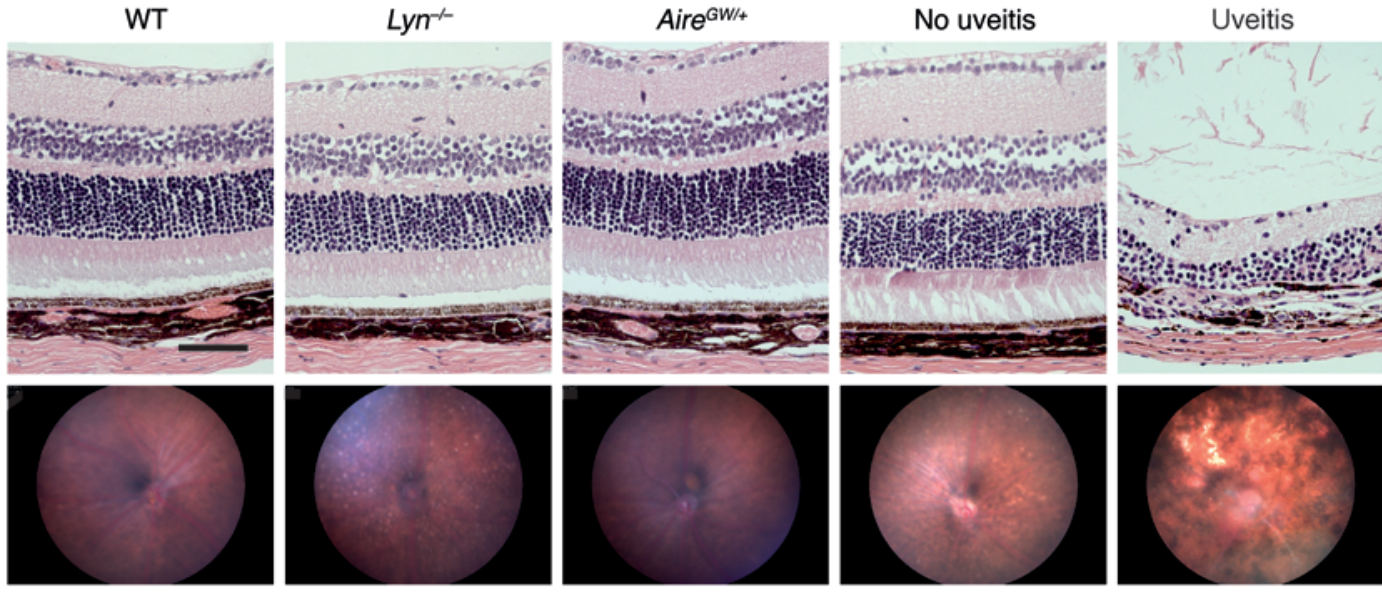

C
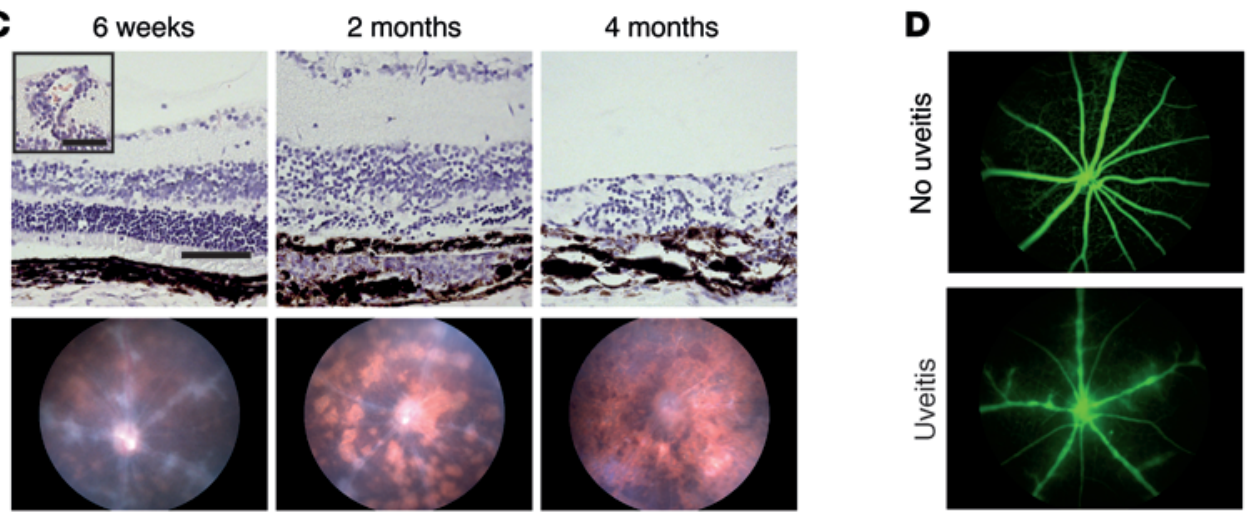

Figure 1. Aire ${ }^{C W /+} \operatorname{Lyn}^{-/-}$double-mutant mice develop progressive posterior uveitis. (A) Eight- to 10-month-old $\operatorname{Aire}^{C W /+} L^{2 y n^{-/-}}(n=10)$, Aire ${ }^{C W /+}(n=4)$, or $\mathrm{Lyn}^{-1-}(n=6)$ mice were analyzed by scoring of H\&E-stained histological sections for presence of inflammatory infiltrates in a panel of organs consisting of eye, lacrimal gland, salivary gland, lung, pancreas, stomach, liver, and sciatic nerve. Pie graphs represent individual mice, with shaded sections indicating the presence of mononuclear infiltrate in the designated organ. The degree of shading reflects the severity of autoimmune damage, as indicated. The characteristics of all 10 double-mutant mice are shown, whereas 3 representative examples of each single-mutant mouse strain are shown. (B) Representative funduscopic images (bottom row) or H\&E-stained retinal sections (top row; original magnification, $\times 20$; scale bar: $100 \mu \mathrm{m}$ ) from 5 -month-old Aire $^{\mathrm{CW/+}} \mathrm{Lyn}^{-/-}$mice with and without uveitis and WT, Aire $\mathrm{CW/+}^{\text {, }}$ and $\mathrm{Lyn}^{-/-}$mice. (C) Time course of histological (top row; original magnification, $\times 20$; scale bar: $100 \mu \mathrm{m}$ ) and funduscopic (bottom row) changes in Aire ${ }^{\mathrm{CW} /+} \mathrm{Lyn}^{-/-}$mice with uveitis. Early changes consisted of swelling of retinal vessels and perivascular exudates (inset; original magnification, $\times 20$; scale bar: $50 \mu \mathrm{m}$ ) leading to infiltration of mononuclear cells, development of inflammatory lesions, and destruction of the photoreceptor layer. Advanced disease was characterized by extensive retinal destruction and scarring. ( $n=3$ mice with uveitis analyzed longitudinally.) (D) Representative fluorescein imaging of retinal vessels in healthy (top) and diseased (bottom) 7-week-old Aire ${ }^{\mathrm{CW} /+} \mathrm{Lyn}^{-/-}$mice. (At least 3 mice per group were analyzed.)

contribute substantially to autoimmune susceptibility for SLE and possibly other autoimmune diseases. An amino acid variant in the transmembrane domain of the inhibitory receptor Fc $\gamma$ RIIb (T232 allelic form; present in approximately $10 \%$ of people of mixed European descent and $20 \%$ to $25 \%$ of Southeast Asians and Africans) excludes this receptor from the lipid raft domains of the plasma membrane and compromises inhibitory function (16), and indi- viduals with the T232/T232 genotype have a 1.7-times relative risk of developing SLE (17). Rarer but exhibiting a larger relative risk are loss-of-function mutations in sialic acid acetylesterase (SIAE), which is critical for the function of CD22, an inhibitory receptor whose function requires LYN; such mutations have been found at elevated frequencies in individuals with SLE, rheumatoid arthritis, and type 1 diabetes (T1D) (18). While most individuals with these 
Table 1. Incidence of uveitis in Aire ${ }^{C W /+} L_{y n}{ }^{-/-}$mice and controls

$\begin{array}{lcc}\text { Genotype } & \text { Uveitis observed } & \text { Incidence (\%) } \\ \text { WT C57BL/6) } & 0 / 50 & 0 \\ \text { Aire }^{C W /+} & 3 / 50 & 6 \\ \text { Lyn }^{-/-} & 0 / 50 & 0 \\ \text { Aire }^{C W /+} \text { Lyn }^{+/-} & 0 / 10 & 0 \\ \text { Aire }^{C W /+} \text { Lyn }^{-/-} & 48 / 100 & 48\end{array}$

Three- to 6-month-old mice of indicated genotypes were screened for uveitis by funduscopy and/or histology. Shown are numbers of mice with disease per total number of mice and resulting incidences.

diseases have normal alleles of SIAE, individuals with loss of function of SIAE have an 8-fold increased risk of developing autoimmune disease. Interestingly, T1D is a quintessential organ-specific autoimmune disease, suggesting that LYN-dependent inhibitory pathways may be important for preventing organ-specific autoimmunity as well as systemic autoimmunity.

To test the hypothesis that a defect in a peripheral tolerance pathway can interact with a defect in central tolerance, we generated $L y n$-deficient mice also harboring a dominant-negative mutation in Aire on an autoimmune-resistant genetic background. Remarkably, these double-mutant mice were found to develop an early-onset, highly penetrant, and highly destructive autoimmune uveitis. Mechanistic analysis of this digenic autoimmune disease model demonstrated that LYN deficiency in DCs results in increased priming in the immunologic periphery of $\mathrm{T}$ cells recognizing an AIRE-regulated retinal self-antigen. This altered priming permitted expansion of an organ-specific population of $\mathrm{T}$ cells that had escaped deletion during development in the thymus because of reduced function of AIRE. Taken together these results demonstrate how defects in distinct genetic pathways that control immune tolerance can synergize to unleash autoimmune disease.

\section{Results}

Aire ${ }^{\mathrm{GW} / \mathrm{+}} \mathrm{Lyn}^{-1}$ double-mutant mice spontaneously develop posterior uveitis. A dominant-negative allele of Aire containing the G228W point mutation when combined with a WT Aire allele results in hypomorphic AIRE function and on the autoimmune-resistant C57BL/6 genetic background leads to a mild autoimmune susceptibility limited to lacrimal and salivary gland infiltrates in Aire ${ }^{\mathrm{GW} / \mathrm{+}}$ mice (19). To determine whether a defect in LYN could enhance this mild susceptibility and affect the disease spectrum, we generated a line of double-mutant Aire ${ }^{\mathrm{GW} /+} \mathrm{Lyn}^{-/-}$mice. Cohorts of double-mutant mice and single-mutant controls were aged for 8 to 10 months and analyzed for the presence of inflammatory infiltrates in organs known to be a target of autoimmune attack in mice completely deficient in Aire $(19,20)$.

Histological sections of lacrimal and salivary glands from Aire ${ }^{\mathrm{GW} /+} \mathrm{Lyn}^{-/}$double mutants revealed lymphocytic infiltrates similar in incidence and severity to Aire ${ }^{\mathrm{GW} /+}$ mice (Figure 1A). Furthermore, infiltrates in the stomach, pancreas, liver, lungs, and sciatic nerve of the double-mutant mice were generally mild or absent (Figure 1A). Two of 10 double mutants had moderate lung infiltrates; however, the sera from these mice were negative for antibodies to BPIFB1, a major lung autoantigen in Aire ${ }^{-/}$mice (data not shown), and similar inflammation was seen in some single-mutant mice (Figure 1A and ref. 21). Surprisingly, histological sections of eyes from 4 of 10 double-mutant mice showed evidence of a severe retinal lymphocytic infiltration, which was absent from the single-mutant mice. Thus, the eye was the only organ from our panel that appeared to be selectively affected in double-mutant mice. In addition, there was no difference in titers of anti-dsDNA IgG antibodies or time of their onset between double mutants and LYN-deficient mice, suggesting that reduced Aire function did not affect lupus-like disease in $\mathrm{Lyn}^{-/}$mice (Supplemental Figure 1; supplemental material available online with this article; doi:10.1172/JCI84440DS1).

We therefore chose to focus our further investigation on retinal disease in the double-mutant mice. The eyes of 5-month-old mice were analyzed by in vivo funduscopic imaging and subsequent histology (Figure 1B). Funduscopy revealed severe inflammation, including multiple retinal lesions with areas of complete retinal destruction, characteristic of advanced uveitis. By histology, disease manifested as extensive mononuclear infiltrates in the retina and choroid, and destruction of photoreceptor outer segments and nuclei. Over the course of the study, a cohort of 100 mice were analyzed at 3-6 months of age, and the incidence of retinal disease was $48 \%$. None of the $\mathrm{Lyn}^{-/}$mice examined developed uveitis, and disease was seen in only $6 \%$ of Aire ${ }^{G W /+}$ mice (Table 1). In addition, ten 4- to 6-month-old Aire ${ }^{G W /+} \mathrm{Lyn}^{+/-}$mice were evaluated by funduscopy and histology and were all found to be free of uveitis, suggesting that haploinsufficiency of LYN was not enough to drive AIRE-dependent uveitis (Table 1).

To gain a better understanding of the dynamics of eye disease, we followed double-mutant mice longitudinally by funduscopy and retinal histology through early, intermediate, and late stages of uveitis (Figure 1C). The first signs of uveitis were typically seen at around 6 weeks of age, with some mice developing infiltrates as early as 5 weeks. Early uveitis was characterized by swelling of retinal vessels and perivascular exudates (Figure 1C, bottom left), with mononuclear cells observed around blood vessels and in the subretinal space (Figure 1C, top left, inset). In mice with earlystage disease, leakiness of retinal vasculature was demonstrated by use of a fluorescein tracer (Figure 1D). These early changes developed into full-blown inflammation around 2 months of age, with multiple retinal lesions consisting of massive mononuclear cell infiltration into the choroid and subretinal space, resulting in obliteration of the rod outer segment layer (Figure 1C, middle). During late stages of disease, complete obliteration of retinal architecture, scarring, and atrophy ensued (Figure 1C, right). The time of onset and dynamics of uveitis in the double-mutant mice were similar to those seen in the Aire ${ }^{-/}$mice (22). Interestingly, the dynamics of uveitis in Aire $\mathrm{GW}^{\mathrm{W} / \mathrm{L}} \mathrm{Lyn}^{-/-}$mice were consistent among multiple cohorts over the course of the study, and mice that were free of uveitis at 8 to 10 weeks of age always remained disease free for as long as they were monitored.

Uveitis in Aire ${ }^{\mathrm{GW} /+} \mathrm{Lyn}^{-/-}$mice is accompanied by an adaptive immune response to IRBP. The uveitis seen in mice completely deficient in Aire is strongly linked to a $\mathrm{T}$ cell response to a retinal autoantigen, interphotoreceptor retinoid-binding protein (IRBP), whose expression in the thymus is controlled by AIRE (23). In 


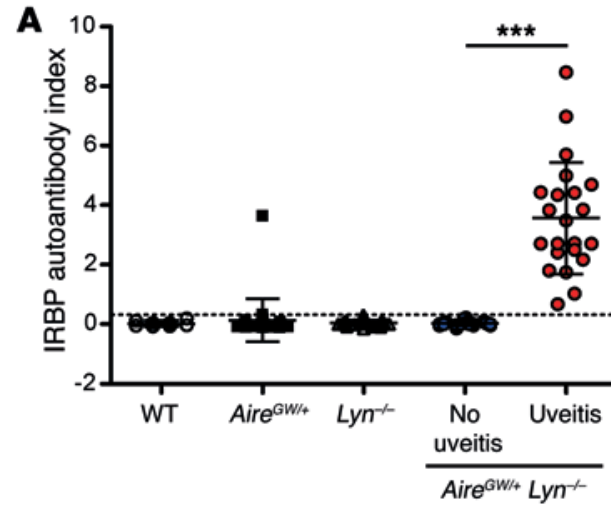

B
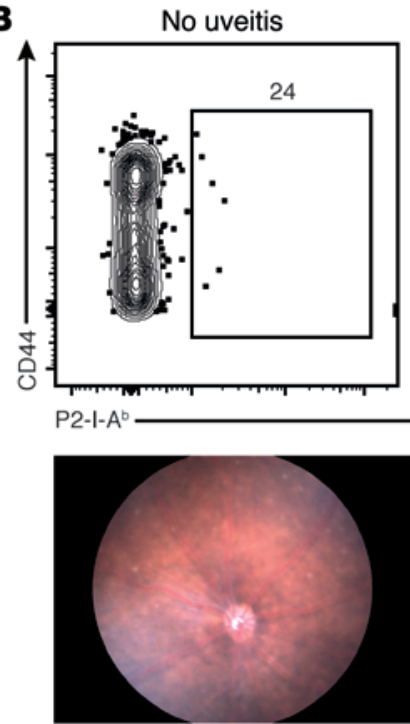

Uveitis
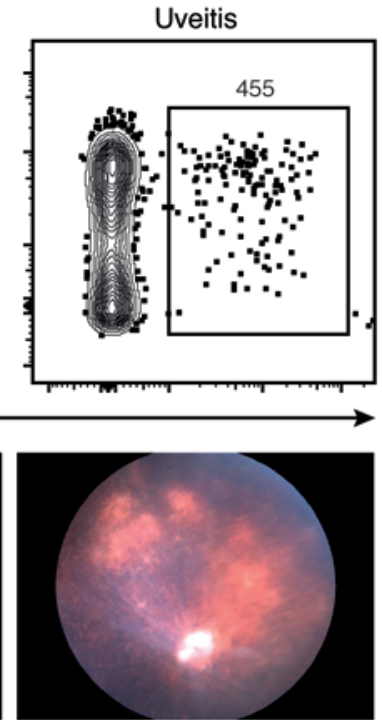

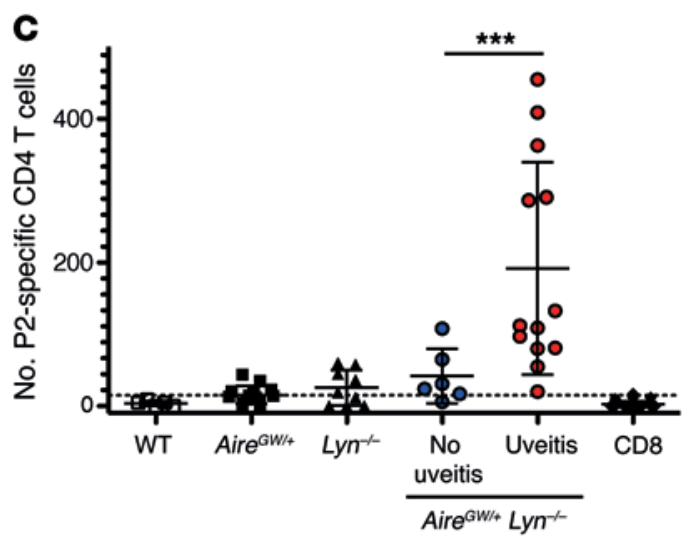

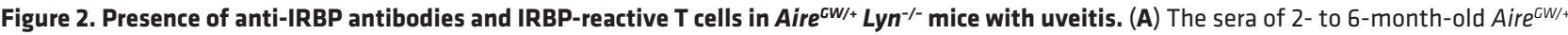
Lyn $^{-/-}$mice with $(n=14)$ and without $(n=9)$ uveitis, or of WT $(n=8)$, Aire ${ }^{C W /+}(n=36)$, or $L y n^{-/-}(n=31)$ mice, were analyzed for the presence of anti-IRBP antibodies with a radioligand binding assay and normalized to a commercially available anti-IRBP antibody to determine autoantibody index (AI). Each dot represents an individual mouse, and the horizontal lines show mean \pm SD. The dashed line represents the limit of detection, calculated as an average of Al values for all WT samples plus 3 SDs. (B) Representative flow cytometric analysis of IRBP P2 tetramer-binding CD4 T cells in pooled spleen and cervical lymph nodes (top) and funduscopy (bottom) from 2- to 5-month-old Aire ${ }^{\mathrm{CW} /+} \mathrm{Lyn}^{-/-}$mice with and without uveitis. Plots were pregated for CD4+ T cells as described in Methods. The calculated total number of tetramer-positive cells in the whole sample is shown on the plot. (C) Numbers of P2-specific CD3+ CD4 T cells gated as in B from individual Aire ${ }^{C W /+} L y n^{-/-}$mice with and without uveitis, and from WT and single-mutant control mice. Each dot represents an individual mouse, and the horizontal lines show mean \pm SD. The dashed line represents the limit of detection, calculated as average number of P2-binding $\mathrm{CD}^{+} \mathrm{CD}^{+} \mathrm{T}$ cells plus $3 \mathrm{SDs}$. Data are pooled from 3 to 5 independent experiments. ${ }^{* *} P<0.001,1$-way ANOVA.

addition, spontaneous uveitis in the Aire ${ }^{G W /+} \mathrm{Lyn}^{-/-}$double-mutant mice started with destruction of the photoreceptor layer (Figure 1C), which is where IRBP functions. Therefore, we decided to examine the adaptive immune responses to IRBP in these mice.

To gain insight into the target of the adaptive autoimmune response in the eye, sera from mice with and without uveitis were immunoblotted against mouse whole eye extracts (Supplemental Figure 2). Sera derived from Aire ${ }^{\mathrm{GW/+}} \mathrm{Lyn}^{-/-}$mice with uveitis but not from unaffected double mutants reacted strongly with a single $150-\mathrm{kDa}$ antigen, which corresponds to the molecular weight of IRBP (23). No other reactivities were detected in this assay, suggesting that there was minimal epitope spreading to other specificities. To examine the magnitude of the anti-IRBP response in a cohort of 5- to 9-month-old double-mutant and control mice, we measured serum IgG binding to radiolabeled mouse IRBP synthesized in vitro (Figure 2A). All double-mutant mice with uveitis had detectable titers of anti-IRBP antibodies. None of the healthy double-mutant mice or control mice had these antibodies, with the exception of a single Aire ${ }^{G W /+}$ mouse, which had disease. Therefore, the presence of anti-IRBP IgGs closely correlated with uveitis.
Uveitis in Aire ${ }^{-/-}$mice is also accompanied by an expansion of $\mathrm{CD}^{+} \mathrm{T}$ cells specific for the P2 peptide, corresponding to amino acids 271-290 of IRBP (24). Importantly, P2-specific T cells are negatively selected in Aire ${ }^{+/+}$mice, and their escape from the thymus occurs in Aire ${ }^{-/}$mice (24). We used a fluorescently labeled $\mathrm{I}-\mathrm{A}^{\mathrm{b}}$ tetramer reagent to detect $\mathrm{P} 2$-specific $\mathrm{CD} 4^{+} \mathrm{T}$ cells within the polyclonal $\mathrm{T}$ cell repertoire of double-mutant mice with and without uveitis. Cells from the eye-draining cervical and submandibular lymph nodes and the spleen of individual mice were pooled, incubated with $\mathrm{P} 2$ tetramer, enriched, and analyzed by flow cytometry. A robust expansion of $\mathrm{P} 2$-specific $\mathrm{CD} 4^{+} \mathrm{T}$ cells was detected in double-mutant mice with uveitis (Figure 2, B and C). Moreover, a majority of tetramer-binding $\mathrm{CD} 4^{+} \mathrm{T}$ cells expressed high levels of the activation marker CD44 (Figure 2B), suggesting previous antigen encounter. These data demonstrate that uveitis in Aire ${ }^{G W /+}$ $\mathrm{Lyn}^{-/-}$mice is accompanied by an adaptive immune response to IRBP, including expansion of AIRE-regulated IRBP-specific CD4 ${ }^{+}$ $\mathrm{T}$ cells in the periphery and production of anti-IRBP antibodies.

Uveitis in Aire ${ }^{G W /+} L^{-1 /}$ mice is accompanied by retinal infiltration of activated $C D 4^{+} T$ cells. We next examined the $\mathrm{T}$ cell response in the retinas of Aire $\mathrm{GWW/}^{\mathrm{G}} \mathrm{Lyn}^{-/-}$mice with uveitis. Con- 
A

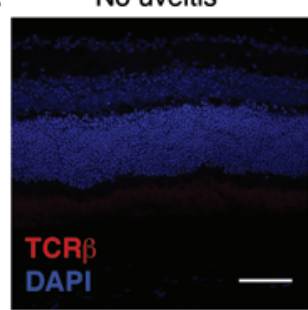

C

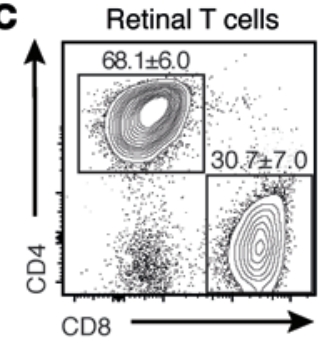

E

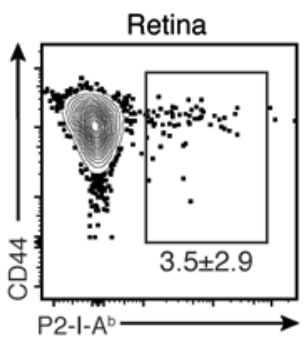

Uveitis

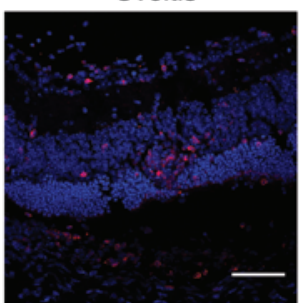

B

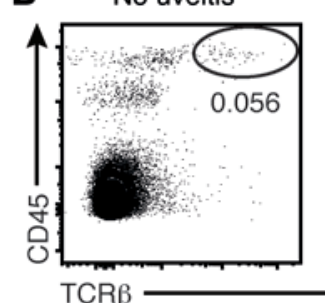

TCR $\beta$
Active uveitis

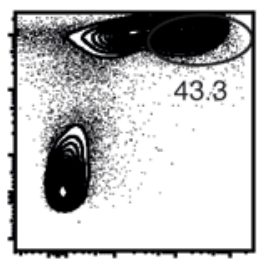

3.3

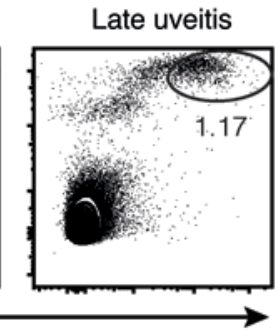

D

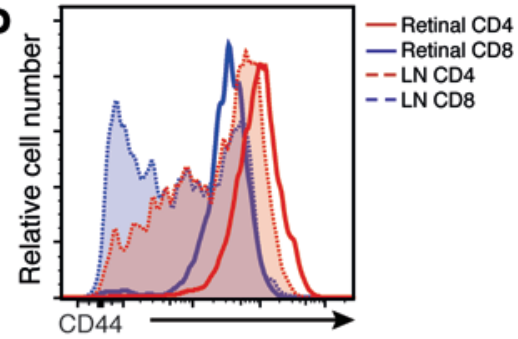

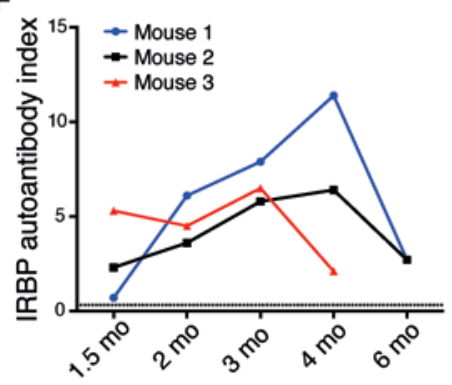

G Draining LNs

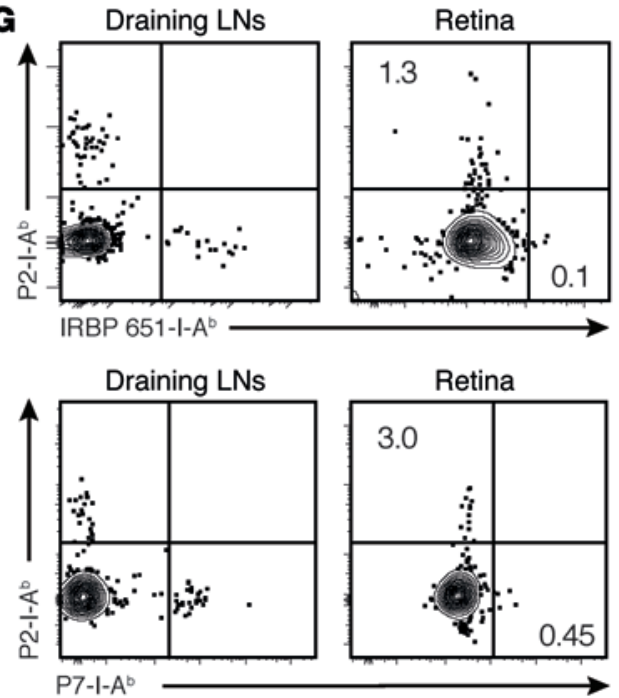

- CD4 T cells

$\triangle \mathrm{CD} 8 \mathrm{~T}$ cells

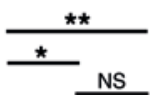

है 힌

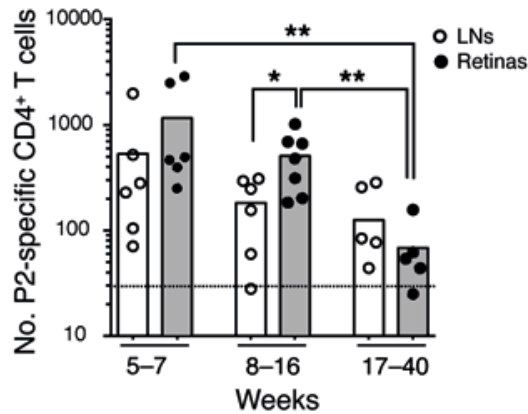

F
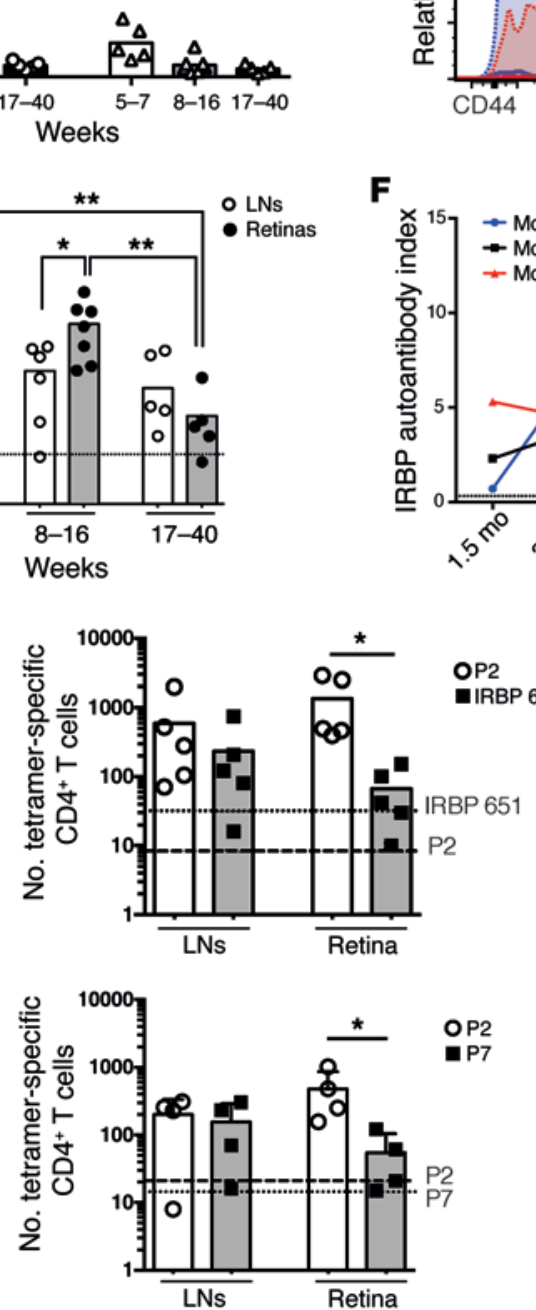

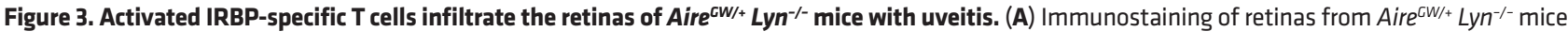
showing infiltration of TCR $\beta^{+}$T cells (red) in mice with uveitis (original magnification, $\times 40$; scale bars: $50 \mu \mathrm{m}$ ). Data are representative of 2 independent experiments. (B-E) Analysis of total and IRBP-specific T cells in mice with uveitis. Plots are representative of 3 independent experiments with $n=3-5$ mice per group; numbers shown indicate percentage of cells in gate. Graphs show data pooled from 3 independent experiments; each dot represents an individual mouse; bars represent mean. (B) CD45 and TCR $\beta$ expression on total live retinal cells. (C) CD4 and CD8 expression on CD45 TCR $\beta^{+}$retinal T cells (left), and numbers of these cells in the retinas of mice of indicated ages (right). (D) CD44 expression on CD4+ and CD8 ${ }^{+}$T cells from indicated tissue. LN, lymph node. (E) Left: Frequency of retinal P2 tetramer-specific CD4+ $\mathrm{T}$ cells from mice in C. Right: Numbers of P2-specific cells in indicated tissue. Dashed line represents the limit of detection. (F) Anti-IRBP antibodies in the serum of Aire ${ }^{\mathrm{CW/+}} \mathrm{Lyn}^{-/-}$mice with uveitis analyzed longitudinally. Dashed line represents the limit of detection. (C) Mice with active uveitis were analyzed for the presence of CD4+ $T$ cells binding either P2 and IRBP 651-670 (“IRBP 651"; top) or P2 and P7 (bottom) epitopes of IRBP in the indicated tissue. Plots show representative costaining; numbers indicate percentage of cells in gate, quantified on the right. Each dot represents an individual mouse; bars show mean. Dashed line represents the limit of detection for the P2 tetramer; dotted line represents the limit of detection for the IRBP 651 (top) or P7 (bottom) tetramer. Data are pooled from 2 independent experiments. ${ }^{*} P<0.05,{ }^{*} P<0.01$, Mann-Whitney test (C, E, and $\left.\mathbf{G}\right)$. 
Table 2. Incidence of uveitis in Aire ${ }^{c W /+} L_{y n^{-/-}}$mice treated with broad-spectrum antibiotics

Cohort

Antibiotic-treated 1

Antibiotic-treated 2

Controls

Uveitis observed
\[ \begin{array}{c}2 / 4 \\ 2 / 3 \\ 4 / 8\end{array} \]

Aire $\mathrm{CW}_{\mathrm{H}+} \mathrm{Lyn}^{-/-}$mice were given a broad-spectrum antibiotic cocktail from birth until 12 weeks of age, while controls received water only. Presence of uveitis was assessed by funduscopy. Shown are numbers of mice with disease per total number of mice and resulting incidences.

focal fluorescence microscopy images of retinal sections from mice at the peak of disease, but not from healthy double-mutant mice, displayed significant $\mathrm{T}$ cell infiltrates in the outer retina and choroid (Figure 3A). To gain insight into the composition and characteristics of this $\mathrm{T}$ cell infiltrate, we isolated healthy or uveitic retinas of Aire ${ }^{G W /+} \mathrm{Lyn}^{-/-}$mice of different age groups, corresponding to early (5-7 weeks), intermediate (8-16 weeks), or late (17-40 weeks) disease based on our funduscopy and histology data (Figure 1C), and analyzed their cellular composition by flow cytometry. As expected, T cells were largely absent from retinas of double-mutant mice without uveitis, whereas diseased retinas manifested a massive influx of T cell receptor- $\beta$-positive $\left(\mathrm{TCR} \beta^{+}\right)$ $\mathrm{T}$ cells, comprising most of the $\mathrm{CD} 45^{+}$infiltrating cells in the retinas during the peak of disease (Figure $3 \mathrm{~B}$ ). The numbers of infiltrating $\mathrm{T}$ cells and other mononuclear cells declined during late stages of the disease. Longitudinal analysis of anti-IRBP antibody titers in double mutants with uveitis revealed a similar dynamic: the titers rose as early as 6 weeks of age, remained high for the duration of active retinal inflammation, and declined at 4-6 months of age (Figure 3F).

Further analysis of the retina-infiltrating TCR $\beta^{+}$cells revealed that $\mathrm{CD}^{+} \mathrm{T}$ cells outnumbered $\mathrm{CD}^{+} \mathrm{T}$ cells approximately 2.5:1 in younger (5- to 16-week-old) mice and to a lesser degree, 1.4:1, in older (16- to 40-week-old) mice with more advanced retinal destruction (Figure $3 \mathrm{C}$ ). Moreover, retina-infiltrating CD4 and CD8 $\mathrm{T}$ cells from mice with active uveitis expressed high levels of the activation marker CD44 (Figure 3D). Interestingly, retina-infiltrating $\mathrm{T}$ cells were more activated, as assessed by CD44 expression, than $\mathrm{T}$ cells from the eye-draining cervical and submandibular lymph nodes isolated from the same animals (Figure 3D). In sum, these data suggest a massive influx of activated $T$ cells into the retina, consistent with a $\mathrm{CD} 4^{+} \mathrm{T}$ cell-dependent autoimmune uveitis.

Retina-infiltrating $\mathrm{CD}^{+} \mathrm{T}$ cells are specific for several epitopes of $I R B P$. To determine whether some of the retina-infiltrating $\mathrm{CD} 4^{+}$ $\mathrm{T}$ cells in the double-mutant mice were specific for IRBP, we performed flow cytometry with the P2 tetramer on retinal single-cell suspensions. A significant fraction of retina-infiltrating $\mathrm{CD} 4^{+} \mathrm{T}$ cells bound to the $\mathrm{P} 2$ tetramer, comprising on average $3 \%$ of total retinal $\mathrm{CD}^{+}{ }^{+} \mathrm{T}$ cells during peak disease (Figure 3E, left). P2-specific cells expressed high levels of the activation marker CD44 compared with the total $\mathrm{CD} 4^{+} \mathrm{T}$ cell infiltrate (Figure $3 \mathrm{E}$, left). Similar numbers of $\mathrm{P} 2$ tetramer-specific $\mathrm{CD} 4^{+}$cells were also seen in the eye-draining lymph nodes (Figure 3E, right). We also used $\mathrm{I}-\mathrm{A}^{\mathrm{b}}$ tetramers to assess the numbers of $\mathrm{CD} 4^{+} \mathrm{T}$ cells recognizing 2 other IRBP epitopes that have been previously analyzed in uveitis in C57BL/6 mice: IRBP 651-670, which has been shown to be highly uveitogenic when used to immunize WT C57BL/6 mice (25), and IRBP 771-790, or P7, which, similarly to P2, is recognized by $\mathrm{CD}^{+} \mathrm{T}$ cells expanded during spontaneous uveitis in peripheral lymphoid organs of Aire ${ }^{-/-}$mice (24). We found that there was robust expansion of both IRBP 651-670-specific and P7-specific $\mathrm{T}$ cells in the eye-draining lymph nodes, and to a lesser extent in the retinas of 6- to 12-week-old Aire ${ }^{\mathrm{GW/+}} \mathrm{Lyn}^{-/-}$mice with active uveitis (Figure 3G). Interestingly, P2-specific CD $4^{+} \mathrm{T}$ cells seemed to be more prevalent in the retinas of mice with uveitis than were $\mathrm{CD} 4^{+} \mathrm{T}$ cells recognizing these other 2 IRBP epitopes.

Depletion of commensal gut bacteria does not prevent uveitis in Aire ${ }^{\mathrm{GW} /+} \mathrm{Lyn}^{-/-}$mice. Signals derived from the microbiota can contribute to activation of innate cells and priming of autoreactive $\mathrm{T}$ cells and have been implicated in a number of autoimmune diseases, including autoimmune uveitis $(26,27)$. Thus, we wondered whether development of uveitis in our model is dependent on the presence of intestinal commensal bacteria. We treated Aire ${ }^{G W /+}$ $\mathrm{Lyn}^{-/-}$mice with a broad-spectrum antibiotic cocktail from birth until 12 weeks of age, at which point they were analyzed by funduscopy for presence of uveitis. Antibiotic treatment was effective in depleting both aerobic and anaerobic bacteria, as assessed by colony-forming unit counts of colonic contents cultured under aerobic or anaerobic conditions (Supplemental Figure 3A); however, there was no difference in the incidence of uveitis compared with that in untreated controls (Table 2). This indicates that signals from commensal bacteria are unlikely to contribute to uveitis development in Aire $\mathrm{GWW}^{\mathrm{W} / \mathrm{Lyn}} \mathrm{LH}^{-/-}$mice.

Deletion of Lyn in DCs combined with reduced AIRE function is sufficient for uveitis. LYN is expressed in most immune cell types other than T cells. Deficiency of LYN in either DCs or B cells is sufficient to lead to spontaneous systemic autoimmunity in C57BL/6 mice $(11,12)$. To gain insight into which cell type promotes uveitis in Aire ${ }^{G W /+}$ mice, we generated Aire ${ }^{G W /+}$ mice carrying a floxed $L y n$ allele and crossed them to $\mathrm{Cre}$-recombinase-expressing lines that delete either in DCs (Cd11c-Cre), in macrophages and neutrophils (LysM-Cre), or in B cells (Mb1-Cre).

Cohorts of mice were aged and evaluated for the presence of uveitis by funduscopic examination starting at 6 weeks of age

Table 3. Incidence of uveitis in Aire ${ }^{C W /+} L y n-D C^{-/-}$mice and controls

\begin{tabular}{|c|c|c|}
\hline Genotype & Uveitis observed & Incidence (\%) \\
\hline$L y n^{f / f f}$ & $0 / 22$ & 0 \\
\hline $\operatorname{Aire}^{C W /+} L y n^{f / f f}$ & $2 / 34$ & 6 \\
\hline Cd11c-Cre Lyn $n^{f / f f}$ & $0 / 30$ & 0 \\
\hline Aire $^{C W /+}$ Mb1-Cre Lyn $n^{f / f f}$ & $0 / 9$ & 0 \\
\hline Aire $^{C W /+}$ LysM-Cre Lyn $n^{f / f f}$ & $0 / 11$ & 0 \\
\hline Aire $^{C W /+}\left[d 11 c-C r e ~ L y n^{f l / f l}\right.$ & $13 / 33$ & 39 \\
\hline
\end{tabular}

Three- to 6-month-old mice of indicated genotypes were screened for uveitis by funduscopy and/or histology. Shown are numbers of mice with disease per total number of mice and resulting incidences. 
A Aire $^{G W /+} L y n^{n I n}$

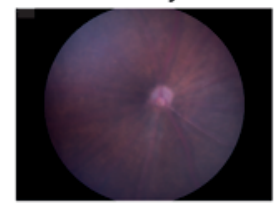

B

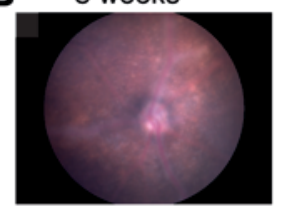

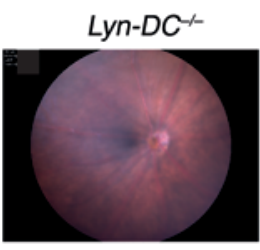

10 weeks

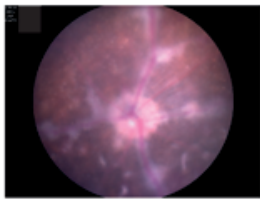

Aire ${ }^{G W /+}$ Lyn-DC+

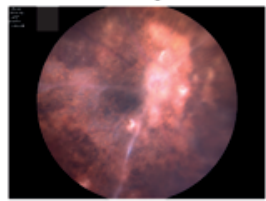

14 weeks

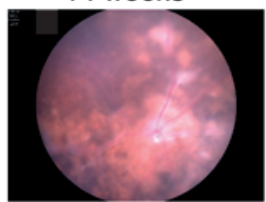

C

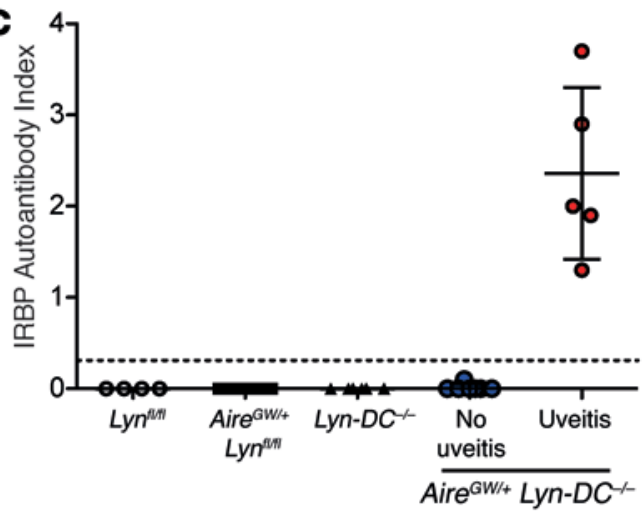

E $\quad$ Aire ${ }^{G W /+}$ Lyn-DC $C^{-}$
D

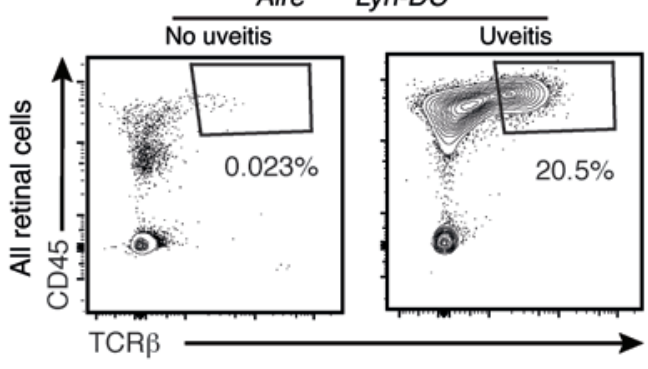

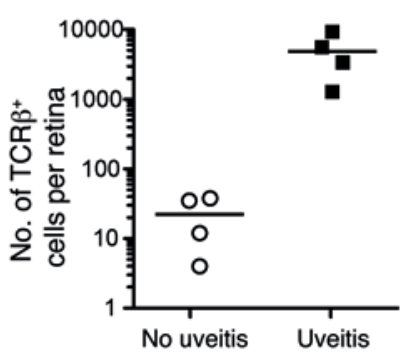
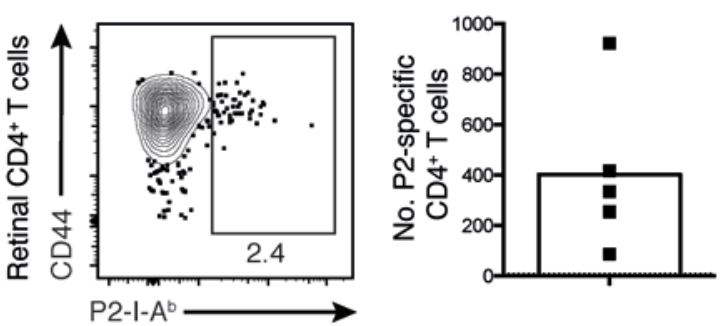
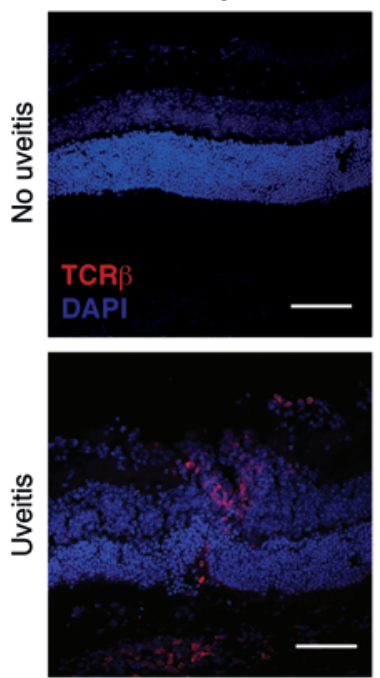

Figure 4. Aire ${ }^{C W /+}$ Lyn-DC-/- mice develop uveitis that is accompanied by an anti-IRBP immune response. (A) Representative funduscopic images of 3 - to 6-month-old mice of indicated genotypes showing retinal disease in a subset of the Aire ${ }^{C W /+} L y n-D C^{-/-}$mice. (B) Time course of funduscopic changes in an Aire ${ }^{C W /+} L y n-D C^{-1-}$ mouse with uveitis (representative of $n=3$ mice with uveitis analyzed longitudinally). (C) Anti-IRBP antibody levels in the serum of 2- to 6-month-old Aire ${ }^{C W /+} L y n-D C^{-/-}$mice with $(n=5)$ and without uveitis $(n=7)$ were measured. Control mice analyzed consisted of $L y n^{f / / f I}(n=8), A i r e^{C W /+}$ $\operatorname{Lyn}^{f l / f l}(n=15)$, and Cd11C-Cre Lyn ${ }^{f l / f l}(n=10)$. Each dot represents an individual mouse; the horizontal lines show mean \pm SD; the dashed line represents the limit of detection. (D) Left set of panels: Plots of retinal cell populations from Aire ${ }^{C W /+} L y n-D C^{-/-}$mice with or without uveitis. Single-cell suspensions from retinas of individual mice were analyzed for CD45+TCR $\beta^{+} \mathrm{T}$ cells (top) and P2 tetramer-specific CD4 T cells (bottom). Numbers shown indicate percentage of cells in gate. Right set of panels: Calculated total numbers of the indicated cells per retina. Each dot represents an individual mouse, and the horizontal lines show mean. The dashed line represents the limit of detection, calculated as in Figure 2. Data are representative of at least 3 independent experiments. (E) Confocal imaging of retinal sections of Aire $\mathrm{CW}^{\mathrm{N}+} \mathrm{Lyn}-D C^{-/-}$mice with or without uveitis showing infiltration of TCR $\beta^{+} \mathrm{T}$ cells (red) in mice with disease (original magnification, $\times 40$; scale bars: $50 \mu \mathrm{m}$ ). Data are representative of 2 independent experiments.

and continuing until 6 months of age. Whereas deletion of Lyn in macrophages and neutrophils or B cells did not lead to disease, deletion of Lyn in DCs resulted in uveitis in 13 of 33 mice (Table 3 and Figure 4A). As with the Aire $\mathrm{GW/H}^{\mathrm{Lyn} /-}$ double-mutant mice, uveitis in Aire ${ }^{G W /+}$ Cd11c-Cre Lyn ${ }^{f l / f l}$ mice (hereafter called Aire $e^{G W /+}$ Lyn-D $C^{-/-}$) occurred at a young age, before overt systemic autoimmunity, and was accompanied by high titers of anti-IRBP antibodies and retinal $\mathrm{T}$ cell infiltrates, including activated $\mathrm{P} 2$ tetramer-specific $\mathrm{CD}^{+} \mathrm{T}$ cells (Figure 4, C-E). These findings indicate that absence of LYN in DCs is sufficient for induction of autoimmune uveitis in Aire ${ }^{G W /+}$ mice.

Interestingly, the onset of uveitis in the Aire $\mathrm{GW/+}^{\mathrm{L} y n-D C^{-/}}$ mice was slightly delayed, with the first funduscopic changes evident at 7-8 weeks of age as opposed to 5-6 weeks of age in the Aire ${ }^{\mathrm{GW} / \mathrm{H}} \mathrm{Lyn}^{-/-}$mice (Figure 4B), indicating that in addition to DCs, Lyn deficiency in another cell type may contribute to development of autoimmune uveitis. Notably, the retina has a resident population of innate immune cells predominantly consisting of microglia but also including DCs $(28,29)$. These cell types are known to express LYN in other tissues; however, expression of LYN in the retina has not been formally demonstrated previously. Using an immunohistochemical approach, we examined retinal sections for LYN expression. Strong LYN expression was detected in the WT retina, but not in the retina of LYN-deficient mice (Supplemental Figure 4). LYN-expressing cells were mostly localized to the inner retina and outer plexiform layer and displayed ramified morphology characteristic of retinal microglia (Supplemental Figure 4). 
A

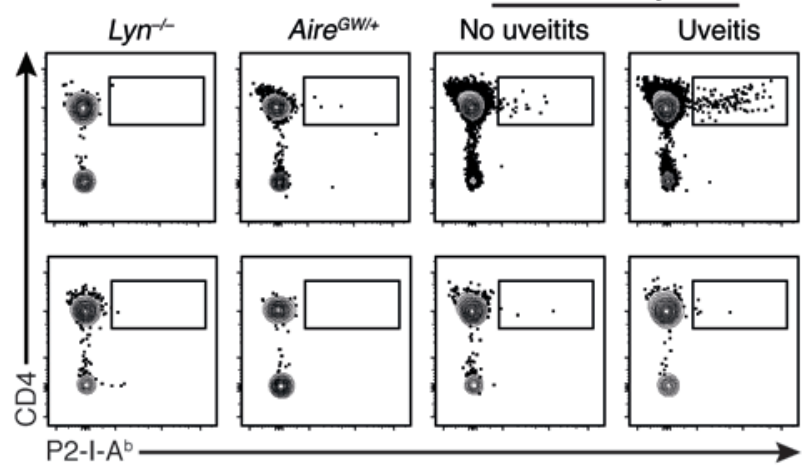

B Draining LNs

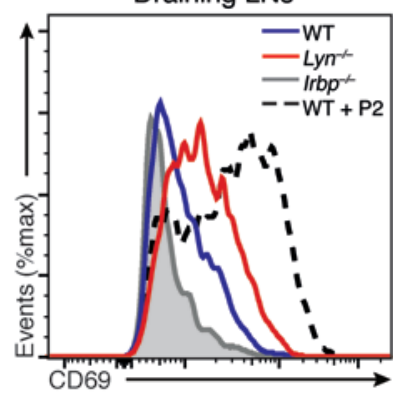

C

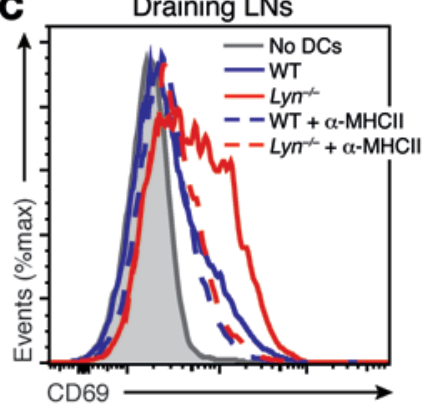

Aire ${ }^{\mathrm{GW} /+} \mathrm{Lyn}^{-1}$

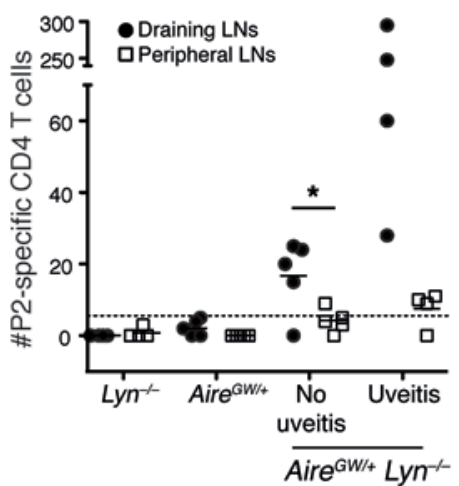

$\mathbf{E}$
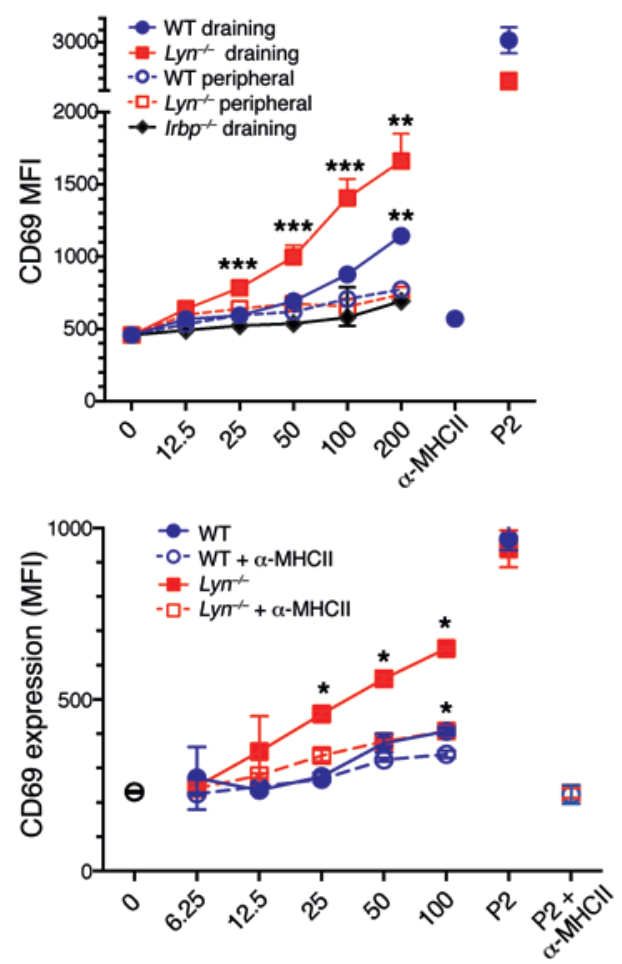

D
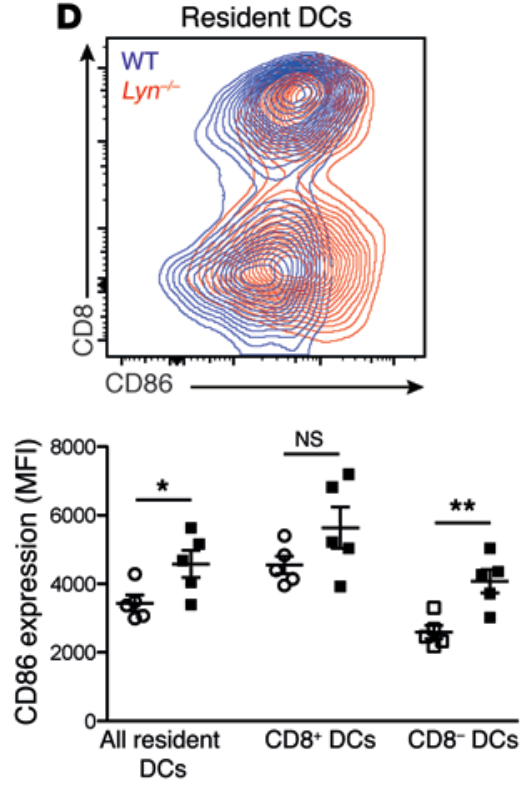

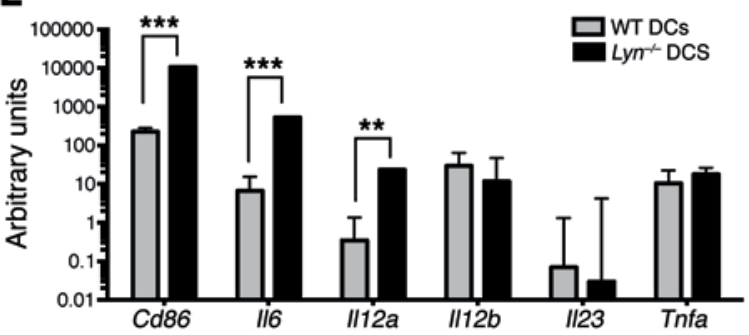

Figure 5. LYN-deficient DCs from the eye-draining lymph nodes present more IRBP and lead to increased priming of IRBP-specific T cells. (A) Left: Representative plots showing P2-specific CD4+ T cells in the eye-draining LNs (top) or in pooled peripheral LNs (bottom) from 15- to 20-week-old mice of indicated genotypes, quantified on the right. Each dot represents an individual mouse; horizontal lines show mean; dashed line represents the limit of detection. Data are pooled from 3 independent experiments. (B and C) Fifty thousand P2-specific T cell hybridomas cocultured overnight with DCs from either eye-draining or peripheral LNs from mice of indicated genotypes were analyzed by flow cytometry for CD69 upregulation. As a positive control, 0.1 $\mu \mathrm{g} / \mathrm{ml}$ P2 peptide was added. (B) Left: CD69 upregulation by hybridomas cocultured with 200,000 eye-draining LN DCs. Right: CD69 expression (mean fluorescence intensity [MFI]) on hybridomas cocultured with varying numbers of DCs from either eye-draining (filled symbols) or peripheral (open symbols) LNs. As a negative control, anti-MHCII blocking antibody was added to hybridomas cocultured with 200,000 WT DCs. (C) Left: Effects of MHCIl blockade on CD69 upregulation by hybridomas cocultured with 100,000 draining LN DCs, quantified over a range of DC concentrations (right). (D) Left: Representative flow cytometry plots showing CD86 and CD8 expression by resident (CD11C $\left.{ }^{+} \mathrm{MHCl}{ }^{\text {int }}\right)$ WT and $\mathrm{Lyn}^{-/-}$cervical LN DCs. Right: CD86 expression on indicated DC populations. Each dot represents an individual mouse; horizontal lines show mean \pm SD. (E) Relative cytokine mRNA expression in WT and $L y n^{-1-}$ sorted resident (CD11C ${ }^{+}$MHCII int) LN DCs, normalized to GAPDH, and shown as arbitrary units. Data are representative of at least 3 (B-D) or 2 (E) independent experiments (4-6 mice per group). ${ }^{*} P<0.05$, Mann-Whitney test $(\mathbf{A}) .{ }^{*} P<0.05,{ }^{* *} P<0.01,{ }^{* * *} P<0.001,1$-way ANOVA (B and $\left.\mathbf{C}\right)$. ${ }^{*} P<0.05,{ }^{* *} P<0.01$, ${ }^{* *} P<0.001$, unpaired 2-tailed Student's $t$ test ( $\mathbf{D}$ and $\left.\mathbf{E}\right)$. 
Increased antigen presentation by $L Y N$-deficient DCs in the eye-draining lymph node leads to expansion of IRBP-specific T cells in Aire ${ }^{\mathrm{GW} / \mathrm{H}} \mathrm{Lyn}^{-/-}$mice. As there were substantial numbers of IRBP $\mathrm{P} 2$ tetramer-binding $\mathrm{CD} 4^{+} \mathrm{T}$ cells in the retinas of Aire ${ }^{\mathrm{GW} /+} \mathrm{Lyn}^{-/-}$ mice with uveitis, we examined their numbers in the eye-draining (cervical and submandibular) or in other peripheral (axillary and inguinal) lymph nodes of individual animals. We found a significant expansion of $\mathrm{P} 2$-specific $\mathrm{CD} 4^{+} \mathrm{T}$ cells in the eye-draining but not in the nondraining peripheral lymph nodes of double-mutant mice with uveitis (Figure 5A). Interestingly, a small but significant population of $\mathrm{P} 2$-specific $\mathrm{CD}^{+} \mathrm{T}$ cells could also be seen in the draining lymph nodes of double mutant mice without uveitis, whereas there was no detectable expansion in either of the singlemutant mice (Figure 5A). This result suggested that there was more IRBP reaching the draining lymph of double-mutant mice without evident inflammation of the retina and/or the LYN-deficient DCs that presented IRBP in the draining lymph were more stimulatory than WT DCs.

To test whether eye-draining lymph node DCs from LYNdeficient mice indeed presented more IRBP, we isolated DCs from either eye-draining or peripheral lymph nodes by anti-CD11c magnetic bead enrichment and examined the ability of DCs from these different sites to stimulate a $\mathrm{T}$ cell hybridoma specific for the P2 peptide of IRBP (24). The DCs from eye-draining lymph nodes of $\mathrm{Lyn}^{-/}$mice were significantly better at stimulating the hybridomas than were WT DCs across a range of DC numbers tested, indicating that more endogenous IRBP was presented (Figure 5B). Importantly, the addition of MHC class II-blocking (MHCII-blocking) antibody largely abolished the increased stimulatory effect of LYN-deficient DCs, indicating that the response involves recognition of peptide/MHCII complexes by the TCR of the hybridoma cells (Figure 5C). As expected, the stimulation of the P2-specific hybridoma was also dependent on the presence of IRBP in the eye-draining lymph nodes, since there was little or no stimulation by DCs from nondraining lymph nodes from the same mice or by draining lymph node DCs from Irbp $p^{-/-}$mice (Figure 5B). Additionally, there was no difference in the hybridoma-stimulatory ability of draining lymph node DCs between young $\mathrm{Lyn}^{-/-}$mice with no antinuclear antibodies and aged mice with high titers of antinuclear antibodies, indicating that increased IRBP presentation in the $\mathrm{Lyn}^{-/-}$mice is likely unaffected by the lupus-like disease in the older mice (Supplemental Figure 5).

To determine whether increased stimulatory capacity of LYN-deficient DCs was due to enhanced processing of IRBP, $\mathrm{Lyn}^{+/+}$or $\mathrm{Lyn}^{-/-}$DCs were given a range of concentrations of exogenous whole IRBP or P2 peptide. Both DCs stimulated the hybridomas equally well, indicating that antigen processing is not augmented in the absence of Lyn (Supplemental Figure 6). Importantly, draining lymph node DCs from $L y n-D C^{-/-}$mice were also better at stimulating the hybridomas than control DCs, indicating that the effect of LYN deficiency was DC-intrinsic (Supplemental Figure 7). Overall, these results suggest that, in the absence of LYN, increased amounts of IRBP are presented by eye-draining lymph node DCs.

We next tested whether there was a difference in activation status between $\mathrm{Lyn}^{+/+}$and $\mathrm{Lyn}^{-/-}$lymph node DC populations by examining surface levels of the CD86 activation marker. LYN- deficient resident CD11b ${ }^{+}$CD8 ${ }^{-}$DCs from the cervical lymph node had significantly higher levels of CD86 expression than their WT counterparts (Figure 5D). Similar upregulation of CD86 was seen on the LYN-deficient CD $11 \mathrm{~b}^{+} \mathrm{CD} 8^{-}$DCs in the nondraining lymph nodes and spleen, whereas CD86 levels were not significantly altered by LYN deficiency for $\mathrm{CD} 11 \mathrm{~b}^{-} \mathrm{CD} 8^{+} \mathrm{DC}$ or migratory DCs (Figure 5D and data not shown). Upregulation of CD86 was also

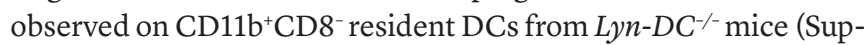
plemental Figure 7). Significantly, CD86 upregulation was not diminished by treatment of $L y n^{-/}$mice with broad-spectrum antibiotics (Supplemental Figure 3). Taken together, these data suggest that an increase in CD86 expression is an intrinsic property of LYN-deficient CD11b ${ }^{+}$DCs.

LYN-deficient DCs from bone marrow and spleen have been shown to produce higher levels of proinflammatory cytokines in vitro in response to stimulation with TLR ligands or cytokines (12, 30). To see whether this is also the case in the steady state, we assessed levels of Il6, Il12a,Il12b, Il23, and Tnfa mRNA ex vivo in unstimulated sorted lymph node DCs. We found that resident DCs from LYN-deficient mice expressed more Il6 and Il12a mRNA as well as more Cd86 mRNA (Figure 5E), in agreement with our flow cytometry data.

\section{Discussion}

Our goal in these studies was to address the mechanism of multigenic susceptibility to autoimmune disease by introducing into mice defects that compromise more than 1 mechanism of immune tolerance and examining how these defects may interact to lead to autoimmunity. For this purpose, we chose to combine a patient-derived dominant-negative allele of Aire, a gene that is important for central tolerance of $\mathrm{T}$ cells to many TSAs, with a complete loss of function of Lyn, which is important for inhibitory receptor function in DCs, B cells, macrophages, and neutrophils. Remarkably, we found that mice with 1 Aire ${ }^{G W}$ allele and 1 Aire $^{+}$ allele, a combination that reduces AIRE function substantially but not completely (31), when combined with a defect in Lyn, develop a highly destructive autoimmune uveitis. On the autoimmuneresistant C57BL/6 background, $\mathrm{Lyn}^{-/-}$mice did not exhibit any eye autoimmunity, and Aire ${ }^{G W /+}$ mice only rarely developed uveitis, so both genetic defects were required to abrogate immune tolerance to eye autoantigens. The contribution of LYN deficiency to this disease was shown to be cell-intrinsic to DCs and likely involves enhanced priming in the eye-draining lymph node of IRBP-specific $\mathrm{T}$ cells that were not tolerized in the thymus because of poor AIRE-induced expression of IRBP. Recent studies have found autosomal dominant mutations in AIRE in a handful of individuals with autoimmune disease $(7,8)$. Individuals with these mutations, which may have functional similarity to the Aire ${ }^{G W}$ allele that we used, exhibit fewer autoimmune features and slower onset when compared with patients with the complete absence of AIRE function. Our findings suggest that additional genetic autoimmune susceptibility alleles may be needed to lead to autoimmunity in these individuals, who may be more common than previously recognized. Moreover, our results provide mechanistic insight into how a defect in $\mathrm{T}$ cell central tolerance and an alteration in peripheral antigen presentation by DCs may synergize to lead to organ-specific autoimmune disease. 
Previous work had established a critical role for AIRE in central tolerance of $\mathrm{T}$ cells that recognize TSAs, including those that recognize the retinal protein IRBP. Here we have shown that Lyn deficiency promotes expansion of IRBP-specific T cells and a severe uveitis in mice with a partial defect in Aire. Unlike Aire $^{-/-}$mice, which completely lack IRBP expression in the thymus, Aire ${ }^{G W /+}$ mice express a small amount of thymic IRBP, which is about one-tenth of the WT levels (19). Thus, we hypothesize that some IRBP-specific T cells escape negative selection in the thymus of Aire ${ }^{\mathrm{GW} /+} \mathrm{Lyn}^{-/-}$mice and can be activated and expanded in the eye-draining lymph node where the LYN-deficient DCs present IRBP peptides. Indeed, there was an expansion of P2-specific $\mathrm{T}$ cells in eye-draining lymph nodes of all double-mutant mice, with a greater expansion in both draining lymph nodes and retinas in mice with disease. Notably, during peak disease, we could also detect expansion of $T$ cells specific for 2 other IRBP epitopes, IRBP 651 and P7, which have been associated with induced and spontaneous uveitis $(24,25)$. Interestingly, there appeared to be more $\mathrm{P} 2$-specific $\mathrm{CD}^{+}{ }^{+} \mathrm{T}$ cells than $\mathrm{T}$ cells specific for these other epitopes in retinas of mice with disease. Moreover, it has been shown that AIRE-directed expression of IRBP in the thymus leads to clonal deletion of P2-specific T cells but not of P7-specific T cells (24). Thus, an attractive hypothesis is that P2-specific T cells initiate disease and that tissue damage leads to epitope spreading to activate IRBP 651- and/or P7-specific T cells, but future experiments will be needed to address the detailed nature of the initiation of uveitis in this new digenic autoimmunity model.

Deletion of Lyn in DCs but not in B cells or myeloid cells was sufficient for disease induction in combination with the dominant-negative allele of Aire. Moreover, DCs from the eye-draining lymph nodes of $L y n^{-/-}$or $L y n-D C^{-/-}$mice were better at stimulating a P2 IRBP-specific T cell hybridoma in the absence of exogenous antigen, indicating that more self-antigen was presented by the DCs in the draining lymph nodes if the DCs were deficient for LYN. This observation likely explains why there was an expansion of IRBP-specific CD4 ${ }^{+} \mathrm{T}$ cells in the eye-draining lymph nodes of the mice without disease. The hyperactive phenotype of LYN-deficient DCs, characterized by increased expression of CD86 and proinflammatory cytokines IL- 6 and IL- 12 by a subset of lymph node DCs (Figure 5, D and E), may have additionally contributed to this expansion. These results indicate that in the Aire ${ }^{\mathrm{GW} /+} \mathrm{Lyn}^{-/-}$mice, there is compromised central tolerance due to reduced AIRE function combined with impaired peripheral tolerance due to absence of Lyn-mediated inhibitory pathways in DCs, leading to the expansion of IRBP-specific T cells, which in about $50 \%$ of the mice go on to infiltrate the retinas and cause autoimmune tissue destruction.

It was surprising that the retina was the only organ severely affected in the Aire ${ }^{G W /+} \mathrm{Lyn}^{-/-}$double mutants and that no synergy was observed for other organs that can be subject to autoimmune attack in mice with complete AIRE deficiency. In part, this restricted failure of immune tolerance may be due to the nature of the autoimmune-resistant C57BL/6 background. C57BL/6 Aire GW/+ $^{\mathrm{W}}$ mice develop infiltrates in the salivary and lacrimal glands only, while on the autoimmune-prone NOD background a number of additional organs are affected despite similarly compromised levels of TSA expression in the thymus (19). This observation suggests that peripheral tolerance mechanisms have a key role in determin- ing whether or not autoreactive $\mathrm{T}$ cells that escape thymic deletion are activated and/or whether their expansion can proceed to autoimmune attack. Interesting in this regard, a previous study has shown that complete AIRE deficiency combined with a defect in $\mathrm{T}$ cell anergy (Cblb) results in autoimmune exocrine pancreatitis (32). We did not see a high-penetrance attack on the exocrine pancreas in the Aire ${ }^{G W /+} \mathrm{Lyn}^{-/-}$mice, suggesting that different combinations of autoimmune susceptibility alleles may differentially sensitize to different forms of organ-specific autoimmunity.

Although we have presented evidence suggesting that LYN deficiency may compromise $\mathrm{T}$ cell peripheral tolerance in a general sense, it is also possible that LYN has a unique role in maintaining tolerance to retinal antigens, perhaps reflecting a role in promoting retinal homeostasis. We found that LYN expression in the retina was localized to ramified cells with typical microglial morphology (Supplemental Figure 4). Retinal microglia can act as antigen-presenting cells, and a subset of them have been shown to express CD11c (29); thus, increased local antigen presentation or increased trafficking of antigen to the draining lymph nodes may play a role in the induction of uveitis in the double-mutant mice. Whether microglial function is altered in LYN-deficient mice, resulting in increased IRBP levels in the eye-draining lymph nodes, is a focus of our ongoing investigation of this new autoimmune model. Notably, Lys $M$-Cre exhibits poor gene recombination efficacy in resting microglia (33), making this strain a poor model to study Lyn deletion in microglia, potentially explaining the lack of uveitis in LysM-Cre Lyn fl/fl mice. In any case, a limited breakdown of tolerance is a feature of the vast majority of patients with autoimmune disease, so understanding the basis of this restriction is an important problem.

The development of uveitis in double-mutant mice followed a time course that closely resembled that of the complete Aire knockout $(20,22)$, with the earliest funduscopic findings appearing at 5 to 6 weeks of age (Figure 1B). In contrast, the first signs of autoimmunity in $\mathrm{Lyn}^{-/-}$mice manifest around 3 to 4 months of age and include progressive increases in antinuclear antibodies, proinflammatory cytokines, and $\mathrm{T}$ cell activation, culminating in severe lupus-like autoimmunity by 8 to 10 months of age (34). Thus, uveitis in the Aire ${ }^{\mathrm{GW} / \mathrm{H}} \mathrm{Lyn}^{-/-}$mouse model precedes the onset of systemic autoimmune disease seen in $\mathrm{Lyn}^{-/-}$mice. Notably, the uveitis that developed in double-mutant mice was always evident by 12 weeks of age, and the remaining $50 \%$ of animals did not develop disease up to 1 year of age (data not shown). Moreover, DCs from LYN-deficient mice with active lupus-like disease did not present increased amounts of IRBP in the eye-draining lymph nodes, as assessed by the use of IRBP-specific T cell hybridomas (Supplemental Figure 5), suggesting that the highly proinflammatory environment in aged $\mathrm{Lyn}^{-/-}$mice was not a major contributor to the onset of autoimmune uveitis.

LYN-deficient DCs are hyperresponsive to stimulation with Toll-like receptor (TLR) ligands, and deletion of MyD88 in a DC-specific or complete Lyn knockout is sufficient to reverse systemic autoimmunity, suggesting that the microbiota may contribute to systemic autoimmunity and inflammatory disease in these mice $(12,30)$. We found that depleting commensal flora in Aire ${ }^{G W /+}$ $\mathrm{Lyn}^{-/-}$mice with a broad-spectrum antibiotic cocktail did not diminish activation of LYN-deficient lymph node DCs or reduce 
incidence of uveitis (Supplemental Figure 4 and Table 2). Breeding these mice under germ-free conditions would be a more stringent test of whether removal of the microbiota affects disease incidence, but the antibiotic treatment we used was highly effective in depleting gut bacteria, suggesting that they are not important for spontaneous development of uveitis. Notably in this regard, MyD88-deficient or germ-free Aire ${ }^{-/-}$mice do not have reduced organ-specific autoimmune disease, suggesting that stimulation of innate immune cells by commensal organisms does not play a major role in activating AIRE-dependent T cell specificities (35).

Our data provide strong genetic evidence that Lyn-deficient DCs are the main cell type contributing to activation of uveitogenic T cells in the Aire ${ }^{\mathrm{GW} /+} \mathrm{Lyn}^{-/-}$mice. Major LYN-expressing cell types include DCs, B cells, and myeloid cells. All 3 are capable of acting as antigen-presenting cells; however, DCs are the most effective cell for priming naive T cells. Deletion of Lyn in either B cells (with Mb1-Cre) or myeloid cells and neutrophils (with LysMCre) did not result in uveitis when combined with the Aire ${ }^{G W /+}$ allele. This result was somewhat surprising, as deletion of Lyn in either B cells or DCs was sufficient for lupus-like disease manifestations, including production of antinuclear antibodies and elevated systemic T cell activation (19). DCs have emerged as key regulators of $\mathrm{T}$ cell tolerance (36), but have also been implicated in the induction of autoimmune disease in a number of mouse models. For example, there is considerable evidence that altering inhibitory or survival signaling in DCs results in systemic autoimmunity and/or inflammatory disease at epithelial barriers. Similar to Lyn, mice with DC-specific deletion of $S h p 1$ or $A 2 O$ have severe lupus-like autoimmune diseases with a strong inflammatory component, whereas mice with a DC-specific deletion of Flip develop severe inflammatory arthritis (37-39). In any case, the results presented here highlight a critical role of Lyn signaling in DCs for promoting tolerance to retinal antigens.

How do LYN-deficient DCs prime IRBP-specific T cells? Important insights into the mechanism were obtained from cocultures of lymph node DCs with an IRBP-specific $\mathrm{T}$ cell hybridoma. Since no exogenous antigen was added to the cultures, the amount of hybridoma stimulation was a readout of the priming ability of lymph node DCs presenting endogenous IRBP. Significantly, DCs obtained from the eye-draining lymph node of WT mice were better at stimulating the hybridomas than their peripheral lymph node counterparts (Figure 5C). This difference was abolished in the presence of an MHCII-blocking antibody (Figure 5C and data not shown), indicating that the stimulatory effect observed was due to the P2 presentation by the DC MHCII complexes. These findings indicate that some retinal antigen was draining to the cervical and submandibular lymph nodes, where it was presented by the DCs. We presume that in an Aire ${ }^{+/+}$ $\mathrm{Lyn}^{-/-}$mouse, the $\mathrm{T}$ cells that are able to recognize the uveitogenic IRBP epitope are absent, and therefore no retinal disease ensues. In contrast, in an Aire-deficient mouse, there are circulating IRBP-reactive cells that can be primed by IRBP-presenting DCs to cause uveitis. Aire $\mathrm{GW}^{\mathrm{W}+}$ mice appear to lie in the middle of this spectrum, since they do not normally develop uveitis on the C57BL/6 background and require an additional "push" from LYN-deficient DCs to develop disease. This interpretation is supported by the fact that DCs from the eye-draining lymph nodes of Lyn-deficient mice were significantly more potent at stimulating the IRBP-specific hybridoma in an MHCII-dependent fashion than were DCs from the eye-draining lymph nodes of WT mice, while no difference was observed in stimulation by DCs from the peripheral lymph nodes (Figure 5, C-E). The IRBP-specific T cell hybridoma was apparently not sensitive to the elevated expression of CD86 by the $\mathrm{Lyn}^{-/-} \mathrm{DCs}$, but it is possible that the in vivo priming of IRBP-specific $\mathrm{CD} 4^{+} \mathrm{T}$ cells was enhanced by both the presence of more IRBP peptide/MHCII complexes and the hyperactive phenotype of the DCs in the draining lymph nodes.

In summary, in order to gain insights into the mechanisms of multigenic autoimmune susceptibility, we created a novel model of digenic autoimmune susceptibility and found that a surprisingly restricted organ-specific autoimmunity emerged from synergy of defects in central $\mathrm{T}$ cell tolerance and inhibitory signaling pathways in DCs. Autoimmune disease is often broadly segregated into organ-specific and systemic autoimmunity. Previous work has suggested that LYN deficiency results in a systemic autoimmune syndrome characterized by anti-DNA antibodies, immune complex deposition in the kidneys, and kidney disease (10). Here we report that LYN deficiency helps provoke an organ-specific autoimmune disease when combined with a partial deficiency in central tolerance to tissue-specific antigens in the thymus. Frequently patients with systemic autoimmune disease like SLE, rheumatoid arthritis, and ankylosing spondylitis will manifest organ-specific autoimmune features like uveitis or thyroiditis $(40,41)$. Our findings here help shed further light on how this may occur through the interplay of innate and adaptive immune tolerance checkpoints. Refined analysis of LYN deficiency demonstrated that it can lead to increased activation of DCs and enhanced antigen presentation, which results in enhanced priming of a small pool of autoreactive $\mathrm{T}$ cells that escape the thymus. Thus, one can envision a model whereby subjects with defects in innate tolerance checkpoints can also develop organ-specific autoimmune features if there is an interaction with an underlying defect in a major adaptive immune tolerance checkpoint like that controlled by AIRE.

\section{Methods}

Mice. Aire ${ }^{\mathrm{GW} / \mathrm{H}}, \mathrm{Lyn}^{-/}, \mathrm{Ly} n^{f / f l}, \mathrm{Cd} 11 \mathrm{c}$-Cre, Mb1-Cre, and LysM-Cre mice were previously described $(12,19,42-44)$. WT mice were obtained from the Jackson Laboratory. All animals were backcrossed at least 10 generations onto the C57BL/6 background and housed in a specific pathogen-free facility at UCSF in accordance with University and NIH guidelines. All mice were screened for the retinal degeneration $8(\mathrm{rd} 8)$ mutation in the crumbs 1 (Crb1) gene using the primers and protocol previously described (data not shown and ref. 45). All strains were negative for the $r d 8$ mutation with the exception of the Mb1-Cre Lyn $n^{f / f l}$ line, which was $C r b 1^{r d s / r d 8}$.

Anti-dsDNA ELISA. Serum titers of anti-dsDNA IgG were determined by ELISA as previously described (46). Antibody titers were normalized to pooled sera from aged MRL/lpr mice run on the same plate.

Western blots. Extracts were made from eyes of WT mice. To screen for the presence of IgG autoantibodies, eye proteins were resolved by SDS-PAGE, transferred to nitrocellulose filters, and incubated with sera from individual mice as previously described (23).

Radioligand binding assay. Full-length mouse IRBP cDNA was transcribed, in vitro translated, and biosynthetically labeled with 
${ }^{35} \mathrm{~S}$-methionine using the TNT Quick Coupled Transcription/Translation System (Promega). Radiolabeled protein was immunoprecipitated with serum samples loaded in triplicate in 96-well PVDF filtration plates (Millipore). Radioactivity retained in the filter was measured using a liquid scintillation counter (1450 MicroBeta Trilux; PerkinElmer). A rabbit polyclonal anti-IRBP IgG antibody was used as a positive control (SC-25787; Santa Cruz Biotechnology). IRBP autoantibody indices for samples were calculated using the following formula: (cpm of unknown - cpm of negative standard) $\div$ (cpm of positive standard - cpm of negative standard) $\times 100$.

Fundus examination. Ocular funduscopy was performed using a Micron III camera (Phoenix Research Labs Inc.). Mice were anesthetized with ketamine $(100 \mathrm{mg} / \mathrm{kg}) /$ xylazine $(10 \mathrm{mg} / \mathrm{kg})$. Tropicamide ophthalmic $1 \%$ and phenylephrine hydrochloride $2.5 \%$ eye drops were applied to relax eye muscles and dilate the pupil. Goniovisc hypromellose $2.5 \%$ was used during imaging and to avoid dryness of the cornea. For the test of blood-retinal barrier integrity, fluorescein angiography was performed immediately after i.p. injection of $20 \mu \mathrm{l}$ of fluorescein sodium $(25 \mathrm{mg} / \mathrm{ml}$ in PBS; Altaire Pharmaceuticals Inc.). Presence of uveitis and disease severity was determined using a previously described grading system (47).

Histology. Organs from mice were harvested and fixed overnight in $10 \%$ formalin, embedded in paraffin, sectioned, and stained with $\mathrm{H} \& \mathrm{E}$. Immune infiltrates of organs were scored as previously described in a blinded fashion (19). Presence of uveitis was determined and scored based on the histological findings.

Immunofluorescence. Whole globes were dissected from the orbit by cutting of the optic nerve and moved to PBS. The clear cornea was removed under a dissecting microscope and the lens and vitreous removed with fine forceps. Eyecups were fixed in $1 \%$ paraformaldehyde for 30 minutes at room temperature followed by washing in PBS 3 times for 15 minutes. Fixed eyecups were embedded in OCT compound, and $20-\mu \mathrm{m}$ sections were cut on a CM3050 S cryostat (Leica Microsystems). Sections were air-dried for 1 hour at room temperature and stained directly or stored at $-80^{\circ} \mathrm{C}$. Dried sections were blocked with BlockAide (Life Technologies) for 1 hour followed by staining with anti-CD3 antibody (rabbit polyclonal; Abcam) diluted in BlockAide at 1:250 overnight in a humidified chamber at $4^{\circ} \mathrm{C}$. Sections were washed with PBS and stained with goat anti-rabbit secondary antibody conjugated to Alexa555 (Life Technologies) diluted in BlockAide at 1:1,000 and DAPI (Life Technologies). Stained retinal sections were then mounted in ProLong Diamond (Life Technologies) and imaged on an SP5 confocal microscope (Leica Microsystems).

Immunohistochemistry. Whole globes were embedded in OCT compound, and $10-\mu \mathrm{m}$ sections were cut and stained as described previously (23) with rabbit anti-mouse polyclonal Lyn antibody (34). Images were acquired with a Zeiss AxioOberver Z1 inverted microscope.

Depletion of intestinal microbiota. Mice were given drinking water containing a broad-spectrum antibiotic cocktail, consisting of vancomycin $(50 \mathrm{mg} / \mathrm{ml})$, metronidazole $(10 \mathrm{mg} / \mathrm{ml})$, gentamicin $(50 \mathrm{mg} /$ $\mathrm{ml})$, colistin $(50 \mathrm{mg} / \mathrm{ml})$, and kanamycin $(50 \mathrm{mg} / \mathrm{ml})$ to deplete both aerobic and anaerobic commensal bacteria as previously described (48). The treatment was given to pregnant dams and continued after weaning until mice were 12 weeks of age.

Tissue preparation and flow cytometry. For tetramer staining, spleen and lymph nodes were dissected, cleaned of fat and fasciae, and transferred to RPMI 1640 medium containing 2\% FBS. Single-cell suspen- sions were prepared by mashing of the cells through a $40-\mu \mathrm{m}$ filter and blocked with anti-mouse CD16/CD32 (24G2) (UCSF Hybridoma Core Facility) and 5\% normal rat serum.

For retinal cell staining, mice were perfused with $20 \mathrm{ml}$ of PBS through the left ventricle of the heart, and both retinas were dissected and transferred to RPMI 1640 medium containing 10\% FBS. Retinas were minced with scissors and digested in the medium containing 0.5 $\mathrm{mg} / \mathrm{ml}$ collagenase D (Roche) and $100 \mathrm{U} / \mathrm{ml}$ DNase (Worthington Biochemicals) for 1 hour at $37^{\circ} \mathrm{C}$. Tissue was dissociated by gentle pipetting and passed through a $70-\mu \mathrm{m}$ strainer. The resulting single-cell suspension was analyzed by flow cytometry using Accucheck counting beads (Invitrogen) to obtain absolute cell counts. To subtract the background numbers of blood-derived retinal $\mathrm{T}$ cells remaining in the vasculature after perfusion, WT mice were injected with fluorescently labeled anti-CD45 antibody i.v. through the retro-orbital venous sinus 10 minutes before perfusion and retinas analyzed by flow cytometry.

For DC sorting and staining, lymph nodes or spleen were digested in RPMI 1640/10\% FBS containing $1 \mathrm{mg} / \mathrm{ml}$ collagenase D and 100 $\mathrm{U} / \mathrm{ml}$ DNase for 1 hour at $37^{\circ} \mathrm{C}$. Immediately after digestion, collagenase was inactivated with $2 \mathrm{mM}$ EDTA, and cells were passed through a $100-\mu \mathrm{m}$ filter and stained.

Antibodies used included CD45 (30-F11; BD Pharmingen), TCR $\beta$ (H57-597; eBioscience), CD4 (GK1.5; Biolegend) CD8 (536.7; Biolegend), CD44 (IM7; Biolegend), B220 (RA3-6B2; eBioscience), F4/80 (BM8; Biolegend), CD11b (M1/70; Biolegend), and CD11c (N418; Biolegend).

Flow cytometry was performed using an LSRII Flow Cytometer (BD Biosciences) at the Flow Cytometry Core at UCSF, and analyzed using FlowJo software (Tree Star Software).

Tetramer analysis. Allophycocyanin-conjugated I-A $\mathrm{b}$ P2 tetramer (QTWEGSGVLPCVG) corresponding to mouse IRBP amino acids 277290 and phycoerythrin-conjugated I-A ${ }^{\text {b }}$ P7 tetramer (SYSSAVPLLCSY) corresponding to mouse IRBP amino acids 771-782 were obtained from the NIH tetramer facility, Atlanta, Georgia, USA. I-A ${ }^{\mathrm{b}}$ IRBP 654-664 tetramer (GAYRTAVDLES) was generated by Moon et al., as described previously (49). Staining of endogenous tetramer-specific T cells was performed as described previously $(24,50)$. Briefly, single-cell suspensions were incubated with tetramer for 1 hour at room temperature, followed by magnetic bead enrichment for tetramer-positive cells. The positively selected cells were stained, and tetramer-reactive cells were gated on $\mathrm{CD}^{+} \mathrm{CD} 4^{+} \mathrm{CD} 8^{-} \mathrm{B} 220^{-} \mathrm{CD}^{-} 1 \mathrm{~b}^{-} \mathrm{CD} 11 \mathrm{c}^{-} \mathrm{F} 4 / 80^{-}$lymphocytes and analyzed by flow cytometry. Counting beads (Accucheck; Invitrogen) were used to obtain absolute cell counts.

For tetramer analysis of infiltrating retinal $\mathrm{T}$ cells, retinal lymphocytes were enriched by centrifugation through a 30\%/37\%/70\% Percoll step gradient and incubated with tetramer for 1 hour at room temperature. Because of the small number of total lymphocytes present in retinas, further enrichment with MACS beads was not necessary, and tetramer-positive cells were analyzed following direct staining of the whole lymphocytic fraction.

Quantitative PCR. Total RNA was extracted from sorted lymph node DCs with a QIAGEN RNeasy Micro kit according to the manufacturer's instructions. RNA was reverse transcribed to generate cDNA using iScript cDNA synthesis kit (Bio-Rad). Cytokine mRNA was quantified using TaqMan Gene Expression Assay probes (Life Technologies) according to the manufacturer's instructions. All samples were normalized to GAPDH, and the values are shown as arbitrary units. 
Antigen presentation assays. Lymph node DCs were enriched by binding to anti-CD11c magnetic beads (Miltenyi), eluted, and plated at serial dilutions in 96-well round-bottom tissue culture plates (Costar) and incubated for 1 hour at $37^{\circ} \mathrm{C}$. P2-binding $\mathrm{T}$ hybridoma cells were previously described (24). Hybridoma cells were labeled with $1 \mathrm{mM}$ carboxyfluorescein diacetate, succinimidyl ester (CFSE SE; Life Technologies) for 10 minutes at $37^{\circ} \mathrm{C}$. The reaction was stopped by addition of complete RPMI 1640 . Fifty thousand hybridoma cells were added per well and cocultured with DCs overnight. For exogenous antigen presentation assays, DCs were incubated with either P2 peptide (Genemed Synthesis) or whole IRBP protein enriched from mouse eye extracts (prepared as described in ref. 24) for 24 hours prior to addition of the hybridoma for 1 hour or 24 hours, respectively. Negative controls included addition of anti-MHCII (I-A/I-E) antibody (clone M5/114.15.2; eBioscience), and culturing of hybridomas in the absence of antigen-presenting cells.

Statistics. Statistical analyses were performed using Prism (GraphPad). Statistical differences between 2 groups were calculated with a nonparametric Mann-Whitney $U$ test or unpaired, 2-tailed Student's $t$ test. Statistical differences between 3 groups or more were calculated with 1-way ANOVA followed by Tukey's post hoc test. An $F$ test was performed to determine whether variances were similar among groups. A $P$ value of less than 0.05 was considered statistically significant. Data are presented as the mean \pm SD where appropriate.

Study approval. All animal studies were approved by the UCSF Institutional Animal Care and Use Committee.

\section{Author contributions}

IP, CAL, DBG, ALD, and MSA designed the study. ALD and MSA supervised the study. IP designed and conducted the experiments with help from CNM, MJ, and KF. IP analyzed the data, and made the figures with help from CNM and MSA. CAL provided mice, and JJM provided the IRBP 654-664 tetramer. IP wrote and ALD and MSA revised the manuscript with contributions from all coauthors.

\section{Acknowledgments}

We thank R. Caspi and M. Mattapallil of the National Eye Institute for assistance with Crb1 genotyping, and helpful discussions, A. Shum of UCSF for running of BPIFB1 immunoassays and helpful discussions, B. Jabri of the University of Chicago for advice regarding the antibiotic treatment regimen, C. Abram, C. Lamagna, L. Lee, and A. Reboldi of UCSF for mice and helpful discussions, and the NIH Tetramer Core Facility for tetramer reagents. This work was supported by grants from the NIH to ALD (AI020038, AI108684), MSA (EY16408, AI097457), CAL (AI065495, AI113272), DBG (EYO19514), Karl Kirchgessner Foundation (DBG), and UCSF graduate division (IP). IP was partially supported by NIH training grants T32AI007334 and T32EY007120. The UCSF Parnassus Flow Cytometry Core and the Diabetes and Endocrinology (DERC) Microscopy Core were supported by the DERC grant NIH P30-DK063720. Additional support was provided by a core grant from National Institute of Health (EYO2162) and Research to Prevent Blindness unrestricted grant to UCSF Department of Ophthalmology.

Address correspondence to: Mark S. Anderson or Anthony L. DeFranco, UCSF Diabetes Center (M.S. Anderson) or Department of Microbiology and Immunology (A.L. DeFranco), 513 Parnassus Avenue, San Francisco, California 94143, USA. Phone: 415.502.8052; E-mail: manderson@diabetes.ucsf.edu (M.S. Anderson). Phone: 415.476.5488; E-mail: Anthony.DeFranco@ ucsf.edu (A.L. DeFranco).

MJ's present address is: Department of Immunology, Genentech Inc., 1 DNA Way, South San Francisco, California, USA.
1. Goodnow CC. Multistep pathogenesis of autoimmune disease. Cell. 2007;130(1):25-35.

2. Cho JH, Gregersen PK. Genomics and the multifactorial nature of human autoimmune disease. N Engl JMed. 2011;365(17):1612-1623.

3. Pillai S. Rethinking mechanisms of autoimmune pathogenesis. JAutoimmun. 2013;45:97-103.

4. Nagamine K, et al. Positional cloning of the APECED gene. Nat Genet. 1997;17(4):393-398.

5. Finnish-German APECED Consortium. An autoimmune disease, APECED, caused by mutations in a novel gene featuring two PHD-type zinc-finger domains. Nat Genet. 1997;17(4):399-403.

6. Anderson MS, Su MA. Aire and T cell development. Curr Opin Immunol. 2011;23(2):198-206.

7. Oftedal BE, et al. Dominant mutations in the autoimmune regulator AIRE are associated with common organ-specific autoimmune diseases. Immunity. 2015;42(6):1185-1196.

8. Cetani F, et al. A novel mutation of the autoimmune regulator gene in an Italian kindred with autoimmune polyendocrinopathy-candidiasis-ectodermal dystrophy, acting in a dominant fashion and strongly cosegregating with hypothyroid autoimmune thyroiditis. J Clin Endocrinol
Metab. 2001;86(10):4747-4752.

9. Xu Y, Harder KW, Huntington ND, Hibbs ML, Tarlinton DM. Lyn tyrosine kinase: accentuating the positive and the negative. Immunity. 2005;22(1):9-18.

10. Scapini P, Pereira S, Zhang H, Lowell CA. Multiple roles of Lyn kinase in myeloid cell signaling and function. Immunol Rev. 2009;228(1):23-40.

11. Lamagna C, Hu Y, DeFranco AL, Lowell CA. B cell-specific loss of Lyn kinase leads to autoimmunity. J Immunol. 2014;192(3):919-928.

12. Lamagna C, Scapini P, van Ziffle JA, DeFranco AL, Lowell CA. Hyperactivated MyD88 signaling in dendritic cells, through specific deletion of Lyn kinase, causes severe autoimmunity and inflammation. Proc Natl Acad Sci US A. 2013;110(35):E3311-E3320.

13. Chan VW, Meng F, Soriano P, DeFranco AL, Lowell CA. Characterization of the B lymphocyte populations in Lyn-deficient mice and the role of Lyn in signal initiation and down-regulation. Immunity. 1997;7(1):69-81.

14. Lu R, et al. Genetic associations of LYN with systemic lupus erythematosus. Genes Immun. 2009;10(5):397-403.

15. Deng Y, Tsao BP. Genetic susceptibility to sys- temic lupus erythematosus in the genomic era Nat Rev Rheumatol. 2010;6(12):683-692.

16. Kono H, et al. FcgammaRIIB Ile232Thr transmembrane polymorphism associated with human systemic lupus erythematosus decreases affinity to lipid rafts and attenuates inhibitory effects on B cell receptor signaling. Hum Mol Genet. 2005;14(19):2881-2892.

17. Willcocks LC, et al. A defunctioning polymorphism in FCGR2B is associated with protection against malaria but susceptibility to systemic lupus erythematosus. Proc Natl Acad Sci US A. 2010;107(17):7881-7885.

18. Surolia I, et al. Functionally defective germline variants of sialic acid acetylesterase in autoimmunity. Nature. 2010;466(7303):243-247.

19. Su MA, et al. Mechanisms of an autoimmunity syndrome in mice caused by a dominant mutation in Aire. J Clin Invest. 2008;118(5):1712-1726.

20. Anderson MS, et al. Projection of an immunological self shadow within the thymus by the aire protein. Science. 2002;298(5597):1395-1401.

21. Shum AK, et al. BPIFB1 is a lung-specific autoantigen associated with interstitial lung disease. Sci Transl Med. 2013;5(206):206ra139.

22. Chen J, Qian H, Horai R, Chan CC, Caspi RR. 
Mouse models of experimental autoimmune uveitis: comparative analysis of adjuvant-induced vs spontaneous models of uveitis. Curr Mol Med. 2015;15(6):550-557.

23. DeVoss J, et al. Spontaneous autoimmunity prevented by thymic expression of a single self-antigen. J Exp Med. 2006;203(12):2727-2735.

24. Taniguchi RT, et al. Detection of an autoreactive T-cell population within the polyclonal repertoire that undergoes distinct autoimmune regulator (Aire)-mediated selection. Proc Natl Acad Sci U S A. 2012;109(20):7847-7852.

25. Mattapallil MJ, et al. Characterization of a new epitope of IRBP that induces moderate to severe uveoretinitis in mice with $\mathrm{H}-2 \mathrm{~b}$ haplotype. Invest Ophthalmol Vis Sci. 2015;56(9):5439-5449.

26. Horai R, et al. Microbiota-dependent activation of an autoreactive $\mathrm{T}$ cell receptor provokes autoimmunity in an immunologically privileged site. Immunity. 2015;43(2):343-353.

27. Kamada N, Seo SU, Chen GY, Núñez G. Role of the gut microbiota in immunity and inflammatory disease. Nat Rev Immunol. 2013;13(5):321-335.

28. Karlstetter M, Ebert S, Langmann T. Microglia in the healthy and degenerating retina: insights from novel mouse models. Immunobiology. 2010;215(9-10):685-691.

29. Lehmann U, Heuss ND, McPherson SW, Roehrich H, Gregerson DS. Dendritic cells are early responders to retinal injury. Neurobiol Dis. 2010;40(1):177-184.

30. Silver KL, Crockford TL, Bouriez-Jones T, Milling S, Lambe T, Cornall RJ. MyD88-dependent autoimmune disease in Lyn-deficient mice. Eur J Immunol. 2007;37(10):2734-2743.

31. Su MA, et al. Defective autoimmune regulator-dependent central tolerance to myelin pro- tein zero is linked to autoimmune peripheral neuropathy. Jimmunol. 2012;188(10):4906-4912.

32. Teh CE, Daley SR, Enders A, Goodnow CC. $\mathrm{T}$-cell regulation by casitas B-lineage lympho$\mathrm{ma}$ (Cblb) is a critical failsafe against autoimmune disease due to autoimmune regulator (Aire) deficiency. Proc Natl Acad Sci U S A. 2010;107(33):14709-14714.

33. Goldmann T, et al. A new type of microglia gene targeting shows TAK1 to be pivotal in CNS autoimmune inflammation. Nat Neurosci. 2013;16(11):1618-1626.

34. Scapini P, et al. Myeloid cells, BAFF, and IFN- $\gamma$ establish an inflammatory loop that exacerbates autoimmunity in Lyn-deficient mice. J Exp Med. 2010;207(8):1757-1773.

35. Gray DH, Gavanescu I, Benoist C, Mathis D. Danger-free autoimmune disease in Aire-deficient mice. Proc Natl Acad Sci U S A. 2007;104(46):18193-18198.

36. Platt AM, Randolph GJ. Does deleting dendritic cells delete autoimmunity? Immunity. 2010;33(6):840-842.

37. Abram CL, Roberge GL, Pao LI, Neel BG, Lowell CA. Distinct roles for neutrophils and dendritic cells in inflammation and autoimmunity in motheaten mice. Immunity. 2013;38(3):489-501.

38. Huang $Q Q$, et al. CD11c-mediated deletion of Flip promotes autoreactivity and inflammatory arthritis. Nat Commun. 2015;6:7086.

39. Hammer GE, et al. Expression of A20 by dendritic cells preserves immune homeostasis and prevents colitis and spondyloarthritis. Nat Immunol. 2011;12(12):1184-1193.

40. Selmi C. Diagnosis and classification of autoimmune uveitis. Autoimmun Rev. 2014; 13(4-5):591-594.
41. Peluso R, et al. Prevalence of thyroid autoimmunity in patients with spondyloarthropathies. JRheumatol. 2011;38(7):1371-1377.

42. Clausen BE, Burkhardt C, Reith W, Renkawitz $\mathrm{R}$, Förster I. Conditional gene targeting in macrophages and granulocytes using LysMcre mice. Transgenic Res. 1999;8(4):265-277.

43. Caton ML, Smith-Raska MR, Reizis B. NotchRBP-J signaling controls the homeostasis of CD8- dendritic cells in the spleen. J Exp Med. 2007;204(7):1653-1664.

44. Hobeika E, et al. Testing gene function early in the B cell lineage in mb1-cre mice. Proc Natl Acad Sci U S A. 2006;103(37):13789-13794.

45. Mattapallil MJ, et al. The Rd8 mutation of the Crb1 gene is present in vendor lines of $\mathrm{C} 57 \mathrm{BL} / 6 \mathrm{~N}$ mice and embryonic stem cells, and confounds ocular induced mutant phenotypes. Invest Ophthalmol Vis Sci. 2012;53(6):2921-2927.

46. Hua Z, et al. Requirement for MyD88 signaling in B cells and dendritic cells for germinal center anti-nuclear antibody production in Lyn-deficient mice. J Immunol. 2014;192(3):875-885.

47. Xu H, Koch P, Chen M, Lau A, Reid DM, Forrester JV. A clinical grading system for retinal inflammation in the chronic model of experimental autoimmune uveoretinitis using digital fundus images. Exp Eye Res. 2008;87(4):319-326.

48. Stefka AT, et al. Commensal bacteria protect against food allergen sensitization. Proc Natl Acad Sci U S A. 2014;111(36):13145-13150.

49. Moon JJ, et al. Naive CD4(+) T cell frequency varies for different epitopes and predicts repertoire diversity and response magnitude. Immunity. 2007;27(2):203-213.

50. Moon JJ, et al. Tracking epitope-specific T cells. Nat Protoc. 2009;4(4):565-581. 